\title{
N8 $8-19123$
}

SHAPE OPTIMIZATION OF THREE-DIKENSIONAL STAMPED AND SOLID AUTOMOTIVE COMPONENTS

M. E. Botkin, R.-J. Yang and J. A. Bennett Engineering Mechanics Department General Motors Research Laboratories Warren, MI 48090-9057

Presented at the 1985 GMR Symposium

$$
\text { and }
$$$$
\text { to be published in }
$$
Symposium Proceedings 
Shape Optimization of Three-Dimensional Stamped And Solid Automotive Components

\author{
M.E.Botkin, R.J.Yang And J.A.Bennett \\ Engineering Mechanics Department \\ General Motors Research Laboratories \\ Warren, MI 48090-9055
}

\title{
ABSTRACT
}

The shape optimization of realistic, three-dimensional automotive components is discussed in this paper. The integration of the major parts of the total process: modeling, mesh generation, finite element and sensitivity analysis, and optimization is stressed. The paper will treat stamped components and solid components separately. For stamped parts a highly automated capability has been developed. The problem description is based upon a parameterized boundary design element concept for the definition of the geometry. Automatic triangulation and adaptive mesh refinement are used to provide an automated analysis capability which requires only boundary data and takes into account sensitivity of the solution accuracy to boundary shape. For solid components a general extension of the two-dimensional boundary design element concept has not been achieved. In this case the parameterized surface shape is provided using a generic modeling concept based upon isoparametric mapping patches which also serves as the mesh generator. Emphasis is placed upon the coupling of optimization with a comercially available finite element program. To do this it is necessary to modularize the program architecture and obtain shape design sensitivities using the material derivative approach so that only boundary solution data is needed. Several realistic component designs will be shown to demonstrate the effectiveness of both capabilities.

$: \quad$ INTRODUCTION

Although structural optimization for sizing varables has been treated extensively in the literature for many 
years [1,2] the problem of designing the shape of a structure for minimum mass is a comparatively new research

topic $[3,4,5]$. Although earlier work $[6,7,8]$ stressed the need for automatically modifying the mesh as the structural shape changes, limitations in the boundary representation and mesh generation aspects kept the capability from being truly automatic. Ultimately, one would like to merely describe the function of the structure to the computer in some convenient manner and then allow the program to automatically produce the optimum design[8]. The basic requirements necessary to do this are as follows: 1) the design model--this describes the shape of the structure, loads and constraints, and the design requirements; 2) the analysis model--the finite element mesh created using fully automatic mesh generation and improved using adaptive mesh refinement; and 3 ) the design modification--a numerical optimization process which iteratively improves the design until convergence to the optimum is obtained. Each of these topics and their implementation into the design program will be discussed.

Previous authors have not addressed the problem of handling the more general case of designing parts which are non-planar. Here the major difficulty is in modeling, in a parametric sense, all of the three-dimensional geometry. To do this it was necessary to extend the existing capability for flat parts using an assembly process of the twodimensional segments. Furthermore, the ability to add curvature to planar segments was provided through the superposition of surface interpolation and transformation capabilities.

For solid components, very little research has been reported $[7,8]$. In this paper emphasis will be placed upon two major aspects which have not been previously treated. The first of these is the efficient calculation of the sensitivities of the displacement and stresses. Secondly, the idea of using one of the many commercially available finite element codes is attractive in order to alleviate the burden of software support of an analysis program sophisticated enough to handle solid models. Both of these issues have been addressed and will be discussed.

The integrated design processes described in this paper will stress the necessity for treating realistic, threedimensional design problems typical of those found in automotive design. For this reason, the shape design element descriptions would be most suited for interfacing with the computer-aided drafting systems on which the geometry is initially created. Additionally, it is absolutely necessary 
to have a capability which is as automatic as possible to free the engineer from the burden of finite element creation and modification and from the equally as great a burden of design modification.

\section{SHAPE OPTIMIZATION OF SHEET METAL PARTS}

Design Model Description

There are a significant number of structural components, such as the typical part shown in Fig. 1, that are produced from a single sheet of uniform thickness material. Using conventional optimization techniques in which element thicknesses are the design variables, little mass reduction can be achieved. To further reduce the mass, the shape of the part and the location of the cutouts must be represented by design variables. The resulting design model must provide the description of the boundary geometry as a function of the design variables and also the finite element structural model. To be most effective in impacting the design process, this information must be efficiently generated from conceptual sketches of the part or obtained through an interface to a computer-aided drafting(CAD) system. For that reason, the approach represented in Figs. 2 and 3 has been chosen. The part shown in Fig. 1 has been modeled in Fig. 2 , using what will be referred to as boundary design elements. As well as associating the boundary witb design variables, the boundary design elements are also used to define the stress constraints. Each boundary design element will be associated with at least one stress constraint which will be computed from the maximum stress of all the finite elements touching that boundary design element. The loads and structural boundary conditions are related to a set of reference nodes which are shown in Fig. 3 as key nodes. This information is in turn automatically transferred to the finite element model once it has been generated.

Mesh Generation

Other work $[6,10]$ has stressed the need for automatically modifying the mesh as the structure changes shape, but it was observed that the commonly used mesh generation techniques based upon coarse isoparametric or transformal mappin $\dot{b}$ patches imposed limitations on the ability to treat large variations in shape. While these techniques do redistribute interior nodes as boundaries move, aspect ratios tend to get objectionably large as the shape becomes significantly different than the initial shape. Mesh grading and solution accuracy are difficult to control as well. 
As an alternative to more traditional mesh generation methods, the use of fully automatic mesh generation based only upon boundary points coupled with adaptive refinement has been proposed[11]. This technique is capable of generating a nearly uniform initial mesh of triangular elements given a set of uniformly spaced boundary points. Thus, as the design changes, uniform triangular meshes can be recreated at any time.

After the design model has been created, the boundaries are automatically discretized into uniform segments called the characteristic length (CL) which is an input value. Automatic triangulation $[12,13]$ is used to create a nearly uniform mesh from the set of boundary points and a set of points placed uniformly throughout the region's interior of approximately the same density as the boundary points. This process of creating the uniform mesh is repeated at each step in the design for which a new boundary description has been generated.

\section{Adaptive Mesh Refinement}

Unlike the design of fixed configuration structures, it is not possible to assure the accuracy of the mesh as the shape changes, since the accuracy of various portions of the mesh will change. The ideas of adaptive mesh refinement can be incorporated to help resolve this difficulty[11].

The mesh refinement process is based upon the variation in strain energy density(SED) as a measure of the error in an element. Once SED variations have been determined for all elements, those elements which have undesirably high values must be selected for subdivision. Elements so selected define refinement regions which can be easily identified by graphical contouring. Since it is not practical from a computational standpoint to consider more than a two-step refinement process during the optimization(one initial and one refined analysis), a concept of multiple refinement regions has been implemented in an attempt to enhance convergence. As an example of the process Fig. 4(a) represents a uniform finite element mesh created using the triangulation technique described previously. Several refinement regions can be specified, as shown in Fig. 4(b), so that the resulting mesh, Fig. 4(c), will be more uniformly graded from coarse to fine. The elements in the region of highest SED variation, represented by the smallest dots in Fig. 4(b), are approximately one-fourth of the size of the initial grid. The region represented by the larger dots contains elements of approximately one-half of the 
initial grid size. As many as six regions can be specified, uniformly graded down to one-eighth of the original grid size. The size of the regions can be varied depending upon the selection of an input parameter.

Obviously, the accuracy due to any refinement is unknown in advance. Although numerous papers have been written

[14,15] on error estimates of total strain energy, this work has not been extended to stresses and displacements. It is desired, for the case of the iterative design process described in this paper, to have a conservative estimate of the converged finite element solution. This information may be obtained in an approximate manner using linear extrapolation, graphically represented in Fig. 5. This is a typical relationship, in the absence of a singularity, between a soluton quantity and mesh size. Several steps of refinement are shown, with each step having reduced the element size in half. The solution will eventually converge to Se and the slope of the curve reflects the rate of convergence. A conservative estimate of the converged solution, represented by points $S i$ and So, may be obtained by extrapolating data points produced by one unrefined analysis and one refined analysis. The extrapolated values will be used as stress constraints.

In order that more realistic three-dimensional plate structures can be analyzed, accurate refinements are necessary for finite elements with bending deformation. In general, refinement works best for conforming elements such as for the constant strain triangle already described. Meshes composed of these elements are always too stiff and solution convergence is predictable as shown in Fig. 5 . On the other hand, meshes composed of nonconforming elements may switch from too stiff to too flexible as the refinement progresses. However, the triangular bending element used in this study [16] has been formulated in such a way as to reduce the degree of nonconformity, and convergence studies show that for uniformly refined meshes the element is always too stiff. Several examples have been presented in Ref. 11 which indicate that although the results are not as predictable as for the constant strain triangle, they are quite satisfactory.

\section{Extension To Nonplanar Parts}

The design process which has been described has been extended in order to handle more realistic stamped sheet metal parts[17]. This was accomplished by treating the part as an assembly of the two-dimensional segments described 
above. Each segment has one completely closed exterior boundary which may contain one or more interior cutouts. Segments may be joined along straight sides to form more complex assemblies. Furthermore, segments may be rotated along the joined edges to form three-dimensional geometry, as shown in Fig 6(a). Because each segment is represented by two-dimensional boundary information only, the addition of surface curvature to a planar segment for added stiffness must be addressed separately. Large curvature, such as a cylinder in Fig. 6(b), is accomplished through the definition of a cylindrical coordinate system for that segment alone. All nodes in that segment are transformed to the new surface. Small curvatures are treated by direct projection as shown in Fig. 6(c). The final assembly process can be seen in Fig. 7 in which all the three-dimensional geometry has been expressed in terms of a small number of parameters which can be treated as design variables.

Interactive Graphics Geometrical Modeling

The need to model more complex geometries makes it obvious that some form of model preparation based upon graphics oriented preprocessing is necessary. Unfortunately, existing finite element preprocessors cannot be used directly, since they offer no means of paramaterizing the shape of the model. Although some of the more recentiy developed modelers do include boundary functions, such as splines, there are no design parameters available externally for use with other programs. Furthermore, since the finite element mesh must change to reflect shape changes, loads and constraints must be associated with boundary functions instead of being directly applied to the finite element mesh, as in the typical modeling system. As a result, a special graphics preprocessor for shape optimization was developed [18], which allows a user to create a paramaterized finite element model. A part is modeled as a collection of planar part segments, which are assembled to form a threedimensional plate structure. Design variables define the shape of each part segment. Loads and constraints are applied to finite element nodes through boundary functions, instead of being applied directly to the nodes.

To begin model preparation, the user first selects the $x$ and $y$ dimensions of the part. Next, commands and cross-hairs are used to create the key nodes and boundary design elements that define the geometry of the part to be optimized. Figure 8 (a) shows the six key nodes needed to define the boundary of a planar triangular bracket. Three exterior key nodes locate the perimeter of the part, while 
three interior key nodes locate an interior cutout boundary. Associated with each key node is a radius, represented as a circle in Fig $8(a)$. The radius, as well as the $x$ and $y$ coordinates, are automatically designated as design variables.

Once the necessary key nodes have been created, the cross-hairs are used to connect the key nodes and create the boundary design elements, as shown in Figure 8 (b). If the same key node is selected twice, a circular arc boundary design element is created. A circular arc element can be used to represent a round boundary, a fillet, or a circular hole. If two different key nodes are selected, the user can choose to connect the two key nodes with either a straight boundary design element or a double cubic boundary design element, as shown in Fig. 3. All design variables specified for a particular element type are automatically assigned when the element is created. Commands are available to link design variables, as required.

Otber commands are available to be used for applying constraints or loads to a given boundary. The terminal cross-hairs are first used to select the boundary to be supported or loaded. The user is then prompted for a constraint type or a load magnitude and direction. The constrained boundaries are indicated by a letter 'C', while the loaded boundaries are indicated with a letter ' $L$ ', as shown in Fig. 8(b). At the time when loads are applied, optimization constraints on displacements can also be specified.

Most real production parts, however, have more complex geometries than these examples. For instance, a common manufacturing operation used to add stiffness to a planar part involves adding a lip, or flange, along the edge of the part. Modeling such a part with a conventional finite element preprocessor is relatively simple, but if the design of the part is to be automated, the geometric model of the part must fulfill the requirements already mentioned.

Commands are available to create multiple part segments as shown in Fig. 7. An additional command can be used specifically for creating flanges, which automates some of the multiple-segment-creation steps.

Fiǵure $\theta$ (a) shows six flanges added around the perimeter of the triangular bracket. A flange is added by using the cross-hairs to locate the portion of the boundary for which a flange is desired. The user is then prompted to specify the flange height at each end. The model is completed by 
specifying the angle that each flange is rotated relative to the base part to form a three-dimensional model. This angle s normally ninety degrees. Each of the six flanges, as well base triangular bracket, is a separate part segment, on which a finite element mesh is generated. Figure $\theta(b)$ shows the assembled finite element model of the triangular bracket, generated from the boundary shape information created with the preprocessor.

\section{THREE-DIMENSIONAL SOLID COMPONENTS}

Only a limited amount of work has been accomplished in three-dimensional shape optimization using solid finite element analysis $[7,8]$. Issues not treated previously will be emphasized in this paper [19]. Because a fully automatic mesh generation scheme which relies only on surface data[20] has yet to be developed, the boundary description format. described for thin parts cannot be implemented for solid three-dimensional parts. Instead, it will be assumed that surface representation and mesh generation will be handled by a generic modeling scheme based upon isoparametric mapping patches described in Ref. 8 and shown for a typical part in Fig. 10.

The two topics which will be addressed are design sensitivities and program architecture. Work in both of these areas were largely driven by the desire to use a variety of structural analysis programs (NASTRAN, ANSYS, ADINA, etc.) to be used with a relatively small amount of additional program development. In this study, NASTRAN was used for analysis.

\section{Design Sensitivity analysis}

The variational design sensitivity theory uses the material derivative concept of continuum mechanics and an adjoint variable method to obtain computable expressions for the effect of shape variation on the functionals arising in the shape design problem. The resulting expressions provide analytical sensitivities of structural response.

The variation of displacement functional $\phi$ with respect to shape change is derived by differentiating the variational equilibrium equation and employing the adjoint variable method, to obtain [21-23]

$$
\partial \psi / \partial b=-\int \Gamma^{\sigma^{j j}}(z) \epsilon^{i j}(\lambda) \Omega^{T} \partial r / \partial b d \Gamma
$$


This equation is an integral along the perturbed boundary in which the required data for evaluation are the stresses from the actual load, $\sigma^{i j}$, the strains from the adjoint load, $\epsilon^{i j}$, the position vector,, $\mathfrak{r}$, and the design variable vector, $b$. It should be pointed out that in Eq. 1 assumptions have been made in the derivation so that the kinematically constrained boundary and loaded boundary are assumed to be fixed, and the variation of the displacement functional is only affected by the normal movement of the boundary of the physical domain. Physically, the adjoint solution required in Eq. 1 is interpreted by applying a unit load at the point where the displacement is of interest.

To see the advantage of Eq.1, a comparison should be made [24] with the well known expression for design sensitivities resulting from the implicit differentiation of the finite element equations

$$
\partial z / \partial b=-K^{-1} \partial K / \partial b z
$$

This equation evaluates the displacement derivative by computing derivatives of the terms of the stiffness matrix. There are two shortcomings to this approach. First, obtaining analytical expressions for the stiffness matrix derivatives is very difficult for boundary movements. These expressions are, in general, different for each element type, thereby requiring special computer code for each different element type. For this reason, a finite difference method is generally used to obtain stiffness derivatives. This usualiy requires a judicious choice of the step size to maintain accuracy. Finally, if it is desired to use a commercial finite program for analysis--for which the source code is not available--it is very difficult to manipulate the stiffness matrices to compute the needed derivatives. For these reasons, Eq. 1 is a more desirable expression for computing displacement sensitivities. The needed stresses and strains can be stored by most programs on files to be used by a post-processing routine to obtain the derivatives.

The stress variation also can be derived to obtain an expression similar to Eq. I, except that the discontinuity of the stresses along the interelemental boundaries has to be properly handled. A characteristic function, which averages stress over a small region, is introduced to treat stress constraints in Refs. 24 and 25 . This approach is similar to using the finite element center as the stress constraint point if the element is chosen as the small region and may lead to a misleading constraint value and may 
result in an undesireable or inaccurate optimum shape if the finite element model is inadequate [25].

An alternative that avoids this problem is to obtain the stress sensitivity at a point, using the definition of stress computation in finite element analysis. The elemental stresses are computed by using the following equation

$$
\sigma=D B z^{e}
$$

where $D$ is the elasticity matrix, B the strain recovery matrix, that contains the derivatives of shape functions, and $z^{e}$ an elemental displacement vector. Differentiating Eq. 3 with respect to the design variables, b, one obtains

$$
\sigma_{i}^{\prime}=\mathrm{D}\left(\mathrm{Bz}_{i}^{\mathbf{e}}+\mathrm{B}_{i}^{\prime} \mathbf{z}^{\mathbf{e}}\right)
$$

where the subscript $i$ with a prime superscript indicates the derivative with respect to the ith design variable. Notice that the first term on the right side of Eq. 4 is only a combination of displacement gradients, and can be obtained by applying a combined adjoint load to the system and using the same formula of Eq. 1 .

The primed matrix of the second term of Eq. 4 can be evaluated from the derivative of the nodal coordinates with respect to shape design parameters [26]. It can be computed analytically or by using a finite difference method. For a linear shape function element, such as constant stress triangular element, the matrix $B^{\prime}$ vanishes, while for a quadratic element, the $B^{\prime}$ matrix is constant. Therefore, the finite difference method is sufficient to evaluate the $B^{\prime}$ matrix, except when a higher order element is used. In this study, analytical derivatives are used for $B$ ' and the eight corner points of the solid element are chosen as the stress constraint points.

\section{Modularized Program Architecture}

It was desired to have a system which uses a commercial finite element code as the analysis capability because of the generally widespead acceptance by the structural analysis community of such codes. A major drawback to achieving this goal is that most commercial finite element codes cannot be used as a subroutine. This problem was addressed by building a system of independently executable program modules in which the overall execution is controlled by job control language. 
The modularized system is comprised of a mesh generator, the finite element code(NASTRAN), the adjoint load and constraints definition program, a design sensitivity analysis module, and an optimization module. Each of those is an independent program and is treated as a module. The flow chart of the system is shown in Fig 11. Initially,. one has to generate a generic model for the structural component, and create a NASTRAN data deck for the NASTRAN run. The whole cycle of the system proceeds as follows: run the NASTRAN code for the actual load; calculate the cost function, constraints, and the adjoint loads using the NASTRAN output; rerun the NASTRAN code for the adjoint loads; and perform the design sensitivity analysis and optimization to obtain a new design. Finally, a new finite element mesh and NASTRAN data deck for the new design are generated.

The MSC/NASTRAN version 63 finite element code is employed for analysis. The new feature of the NASTRAN data base is used to save computing time for reanalysis of the adjoint loads. This data base, created by tbe first NASTRAN run, preserves the stiffness and boundary condition information and results in easier input data preparation and less computing time for the reanalysis. The displacements, stresses, and geometric information that are needed for design sensitivity calculation are obtained by using an ALTER feature in NASTRAN to write that information on a file for postprocessing.

The ADJLOD module(Fig. 11) is used to define the cost function and constraints for the design problem, and to calculate the adjoint loads for the constraints which are active or violated. The displacements, stresses, and geometric information from the NASTRAN output are first read to define the constraints for the structural component. A NASTRAN deck containing the adjoint loads is then created for reanalysis.

The SENSTY module(Fig. 11) performs the design sensitivity analysis for the cost and the active constraints, and then performs the optimization process by calling the optimizer(CONMIN[27]) as a subroutine. Before executing the module, the NASTRAN output files for the actual load and the adjoint loads should be available. The module then changes the design and creates new input data for the MESHGN module which will generate a new mesh and a new NASTRAN data file for the next design iteration, if necessary. 


\section{DESIGN EXAMPLES}

Three-Dimensional Sheet-metal Part

Figure 12 shows the initial shape and dimensions of a realistic design example of a sheet metal part [17]. The model was initially created in two dimensions and then segments 2 and 4 were transformed into the third dimension. Structural boundary conditions were imposed around the holes labelled $C$ and $D$. Loads $P 1$ and $P 2$ were applied at hole $A$ in the $y$ and $z$ directions, respectively. Load P3 was applied at hole $B$ in the $y$ direction. The design criteria were a stress limit on all boundaries and a displacement limit at hole $A$. CL was chosen to be $0.80 \mathrm{~cm}$ for the initial mesh.

The current model is similar to an earlier part [17], except that flanges on the new model add seven flange design variables to the problem. The locations of these design variables are shown in Fig. 13. A total of nineteen design variables were used to parameterize the part's shape. Figure 14 shows the initial, unrefined finite element mesh.

This part was modeled to determine how the program would reduce the mass and tailor the flanges, subject to a displacement constraint. A displacement constraint was applied to the hole $A$, such that the displacement of the point was limited to 1 millimeter in the -2 direction. Figure 15 shows the initial and final part designs. The program removed material from the interior cutouts on the base triangular part segment and the cylindrical part segment. A small amount of in-plane curvature was added along the edges of the triangular part segment to which flanges are attached. The flange heights were reduced to less than half the initial values everywhere except along the upper edge of the triangular part segment. The flange heights along this edge are controlled by flange design variables 3 and 4 , as shown in Fig. 13. This edge serves as the primary load path for the structure, since it transfers the load from the tip of the triangular part segment to the support points. As a result, one would expect the flange along this edge to be the most important in maintaining the stiffness of the part. The flange design variable values for the initial and final designs are given in Table 1.

Figure 16 shows the design history for this part. A design variable move limit of five percent was used for the first ten steps, followed by a move limit of 2.5 percent for the last fourteen steps. The characteristic length was reduced from .8 to .6 in the last four steps to obtain more 12 
accurate displacement values in the unrefined analyses. The reduction of the characteristic length eliminated design oscillations that emerged once the displacement constraint became active. The initial unrefined finite element mesh included 3000 degrees of freedom, while the initial refined mesh contained 4000 degrees of freedom.

Finally, some comments are in order concerning the results. First, the design history (Fig. 16) does not show traditional convergence behavior. The optimizer was turned off when it was felt that further mass reduction would require an excessive amount of computer time. Second, one might question the finite element accuracy in the flange areas. Constant strain elements were used, and only one or two elements were used to span the depth of each flange in the unrefined mesh. Bending of the flanges could result in stress variations that would not be picked up by so few constant strain elements. For this reason, the automatic mesh refinement technique described above was used to minimize this error.

Table 1. Design Variables for Transmission Bracket

\begin{tabular}{|c|c|c|c|c|}
\hline No. & initial & final & lower bound & upperbound \\
\hline $\begin{array}{l}1 \\
2 \\
3 \\
4 \\
5 \\
6 \\
6 \\
7\end{array}$ & $\begin{array}{l}2.12 \\
1.50 \\
1.50 \\
1.50 \\
2.12 \\
1.50 \\
1.50\end{array}$ & $\begin{array}{l}0.91 \\
0.68 \\
0.89 \\
0.89 \\
0.96 \\
0.68 \\
0.67\end{array}$ & $\begin{array}{l}0.5 \\
0.5 \\
0.5 \\
0.5 \\
0.5 \\
0.5 \\
0.5\end{array}$ & $\begin{array}{l}3.0 \\
3.0 \\
3.0 \\
3.0 \\
3.0 \\
3.0 \\
3.0\end{array}$ \\
\hline
\end{tabular}

Three-Dimensional Solid Part

An idealized engine connecting rod, which connects the crank shaft and piston pin of an engine and transmits an axial compressive load during firing and a tensile load during the intake cycle of the exhaust stroke, is employed as the example[19]. Shape optimization of similar components have been studied by Yoo et al. [28] and Yang et al. [29] assuming a plane stress state. However, a fully threedimensional shape optimization for the connecting rod is still not available in the literature.

Figure 17 shows the generic model for the connecting rod. For simplicity, the right hole of the connecting rod 
which connects the piston pin is fixed to eliminate rigid body motion; and the arbitrarily selected pressure of 3000 $\mathrm{MPa}$ is applied to the left hole, from 0 to 90 degrees, to simulate the firing forces. The von Mises stress constraint is imposed at each node in the finite element model of the connecting rod. The critical yield stress used for analysis is chosen as $3000 \mathrm{MPa}$. Young's modulus and Poisson's ratio are $10.0 \times 10 E 6 \mathrm{MPa}$ and 0.3 , respectively. The numerical data were selected to demonstrate the use of the system and are not representative of a specific production part.

Using the symmetrical conditions, only a quarter of the structure needs to be analyzed. The design variables are shown in Fig. 17. In this model, 8 design variables are chosen; 5 parameters define the shape of the shank and neck regions, 2 are the outer radii of the right and left boles, and 1 parameter defines the height of the web. The finite element model, as shown in Fig. 18, contains 105 solid(20 node) elements, 828 nodal points, and 2126 degrees-offreedom.

The initial values of the design variables are shown in Table 2. Initially, the volume is $15686.7 \mathrm{cu} \mathrm{mm}$ with no stress violation. After 20 design iterations, it is reduced to $7217.8 \mathrm{cu} \mathrm{mm}$ with no stress violation. The final design variables and the final shape are shown in Table 2 and Fig. 17 , respectively. Figures 19 and 20 show the design histories for the cost and the maximum constraint values, respectivily, of the idealized connecting rod. In Fig. 19, one observes that the convergence rate is reasonably good. From design iterations 10 to 17 , the optimizer tries to force the design into the feasible region. The slow correction for stress violation shown in Fig. 20 may result from Taylor's series expansion approximation for functions.

Table 2. Design Variables for Engine Connecting Rod

$\begin{array}{lcccc}\text { No. } & \text { initial } & \text { final } & \text { lower bound } & \text { upperbound } \\ 1 & 10.956 & 12.512 & 0.1 & 100.0 \\ 2 & 6.37 & 2.8478 & 0.1 & 100.0 \\ 3 & 3.9667 & 1.4220 & 0.1 & 100.0 \\ 4 & 3.0024 & 1.0964 & 0.1 & 100.0 \\ 5 & 3.2711 & 1.2733 & 0.1 & 100.0 \\ 6 & 6.8156 & 7.2219 & 0.1 & 100.0 \\ 7 & 31.271 & 26.461 & 24.0 & 100.0 \\ 8 & 17.553 & 13.300 & 13.3 & 0\end{array}$




\section{SUMMARY}

An integrated approach to the shape design problem has been described for sheet-metal parts in which the problem description is stated in a simple format, the finite element mesh is generated automatically, and its accuracy is improved by adaptive mesh refinement. Non-planar structures can be treated using an assembly process of two-dimensional segments in such a way that all three-dimensional geometry is expressed in terms of a relatively small number of parameters. Surface curvature variations can be added to the planar sub-assemblies through the superposition of a variety of surface transformation and mapping options. All of the geometric problem description has been formulated in such a way that it is particularly suitable for interface to modern CAD systems.

It was found that for the design problem in which the boundaries of the part are moving, the accuracy of the finite element mesh must be continuously assessed and updated. Strain energy density variations within an element were used as a measure of error. Elements with errors greater than a specified value in an unrefined analysis were refined by adding nodes, and a new mesh was created using automatic triangulation. Results of the refined analysis were combined with the unrefined results to compute stress intensification factors which were used to approximate a refined solution for intermediate designs in which refinement did not take place.

The development of a modular computer program for the shape optimization of three-dimensional solid components is also discussed. The program uses NASTRAN for analysis and CONMIN for optimization. Since design sensitivities with respect to shape variables are not available in NASTRAN, a module had to be written to obtain these sensitivities which is based upon the material derivative concept applied to the variational state equation. Parameterized surface definitions and the finite element mesh were obtained from a module based upon generic modelling concepts. Each program module is a separately executable program but all modules can be executed sequentially using Job Control Language. A realistic design example has been provided to demonstrate the capabilities of the program.

In general, it has been shown that it is possible to automate the structural design process for determining the shape of quite complicated three-dimensional components 
through the integration of a parameterized geometric description, automatic mesh generation, finite element analysis, design sensitivity analysis, and optimization. The resulting capabilities eliminate the need for tedious data transfer inherent in existing trial and error design approaches as well as eliminating many of the repetitive steps involved.

\section{REFERENCES}

1. Schmit, L. A., "Structural Synthesis by Systematic Synthesis", Proc. 2nd Conf.on Electronic Computation ASCE, New York, 105-122(1960).

2. Vanderplaats, G.N., "Structural Optimization - Past, Present, and Future" ÁLA Journal, Vol. 20, No. 7, 892 1000 (1982).

3. Zienkiewicz, O. C., and Campbell, J. S., "Shape Dptimization and Sequential Linear Programing," Chap. 7 in OPTIMUM STRUCTURAL DESIGN, edited by R. H. Gallagher and 0 . C. Zienkiewicz, John Wiley \& Sons, New York(1973).

4. Haug, E. J., Choi, K. K., Hou, Y. M., and Yoo, Y. M. "A Variational Method for Shape Optimal Design of Elastic Structures," OPTIMAL STRUCTURAL DESIGN II, (Ed. R. H.

Gallagher), Wiley, New York(1983)

5. Haftka,R.T. and Gandhi,R.V.,"Structural Shape Optimization-A Survey", The 26th AIAA SDM Conference, CP No. 85-0772,617-628(1985).

6. Botkin, M. E.,"Shape Optimization of Plate and Shell Structures," AIAÁ Journal, Vol. 20, No. 2, 268-273(1982).

7. Imam, M. H., "Three-Dimensional Shape Optimization," International Journal for Numerical Methods in Engineering, Vol. 18, 661-673(1882).

8. Imam ,M. H., "Minimum Weight Design of 3-D Solid Components", Proceedings of the 2nd ASME Computers in Engineering Conference, Vol. 3, 119-126(1882).

9. Bennett, J. A., and Botkin, M. E., "Structural Shape Optimization with Geometric Problem Description and Adaptive Mesh Refinement", AIAA Journal, Vol.23, No.3, 458-464 (1985). 
10. Braibant, V. and Fleury, C., "Shape Optimal Design Using B-Splines", Computer Methods in Applied Mechanics and Engineering, Vol. 44, 247-267(1884).

11. Botkin, M. E., "An Adaptive Finite Element Technique for Plate Structures", Technical Note, AIAA Journal, Vol.23,

No.5, 812-814(1985).

12. Cavendish, J. C., "Automatic Triangulation of Arbitrary Planar Domains for the Finite Element Method," International Journal for Numerical Methods in Engineering, Vol. 8, 678686,1874 .

13. Frey, W.H. and Cavendish, J.C., "Fast Planar Mesh Generation Using the Delaunay Triangulation",Presented to the Society for Industrial and Applied Matbematics Meeting, Seattle, WA, July 16-20(1984).

14. Babuska, I., and Rheinbolt, W. D., "Adaptive Approaches and Reliability Estimates in Finite Element Analysis,"

Computer Methods in Applied Mechanics and Engineering, No. $17 / 18,519-540$ (1978).

15. Shephard, M. S., "Finite Element Grid Optimization with interactive Computer Graphics," Ph.D Thesis, Department of Structural Engineering, Cornell University(1978).

16.Conner, J. J. and Will, G., "A Triangular Flat Plate Bending Element," M.I.T., Department of Civil Engineering, Report TR-68-3,Cambridge, MA(1868).

17. Botkin, M. E., and Bennett, J. A., "Shape Optimization Of Three-Dimensional Folded Plate Structures," 1884 AIAA SDM Conference, CP No. 84-0856, Palm Springs, CA., May 14-16, (1884).

18. Botkin,M.E. and Gressel,G.S.,"Shape Optimization of Sheet Metal Components With Flanges", to be presented at the 6th SAE international Vehicle Structural Mechanics Conference, Detroit, Michigan, April 22-25(1986).

19. Yang, R. J., and Botkin, M. E., "A Modular Approach For Three-Dimensional Shape Optimization Of Structures", to be presented at the 27th AIAA SDM Conference, San Antonio, Texas, May 19-21(1986).

20. Yerry, M. A., and Shephard, M. S., "Automatic Threedimensional Mesh Generation by the Modified-octree Technique," International Journal for Numerical Methods in Engineering, Vol. 20,No.11, 1965-1890(1884). 
21. Haug, E. J., Choi, K. K., Hou, J. W. , and Yoo, Y. M., "A Variational Method for Shape Optimal Design of Elastic Structures," New Directions in Optimum Structural Desiga, Ed. E. Atrek et al., Wiley, New York(1984).

22. Choi, K. K. and Haug, E. J., "Shape Design Sensitivity Analysis of Elastic Structures," Journal of Structural Mechanics, 11(2), 231-269(1983).

23. Haug, E. J., Choi, K. K., and Komkov, V., Design Sensitivity Analysis of Structural Systems, Academic Press, (1885).

24. Yang, R. J., and Botkin, M. E., "The Relationship Between the Variational Approach and the Implicit Differentiation Approach to Shape Design Sensitivities," presented at the 1885 AIAA SDM Conference, CP No. 85-0774, Drlando, Florida, April 15-17, (1985).

25. Yang, R. J., Choi, K. K., and Haug, E. J., "Numerical Considerations in Structural Component Shape Optimization," ASME Journal of Mechanisms, Transmissions, and Automation in Design, paper No. 84-DET-219(1884).

26. Ramakrishnan, C. V. and Francavilla, A., "Structural Shape Optimization Using Penalty Functions," Journal of Structural Mechanics, 3(4), 403-422(1974-1875).

27. Vanderplaats, G., "CONMIN - A Fortran Program for Constrained Function Minimization User's Manual," NASA, TM X $62,282(1973)$.

28. Yoo, Y. M., Haug, E. J., and Choi, K. K., "Shape Optimal Design of An Engine Connecting Rod," ASME Journal of Mechanism, transmissions, and Automation in Design, Vol. $106,415-489$ (1884).

29. Yang, R. J., Choi, K. K., and Haug, E. J., "Finite Element Computation of Structural Design Sensitivity Analysis," Report CCAD No. 84-3, The University of Iowa, (1984). 


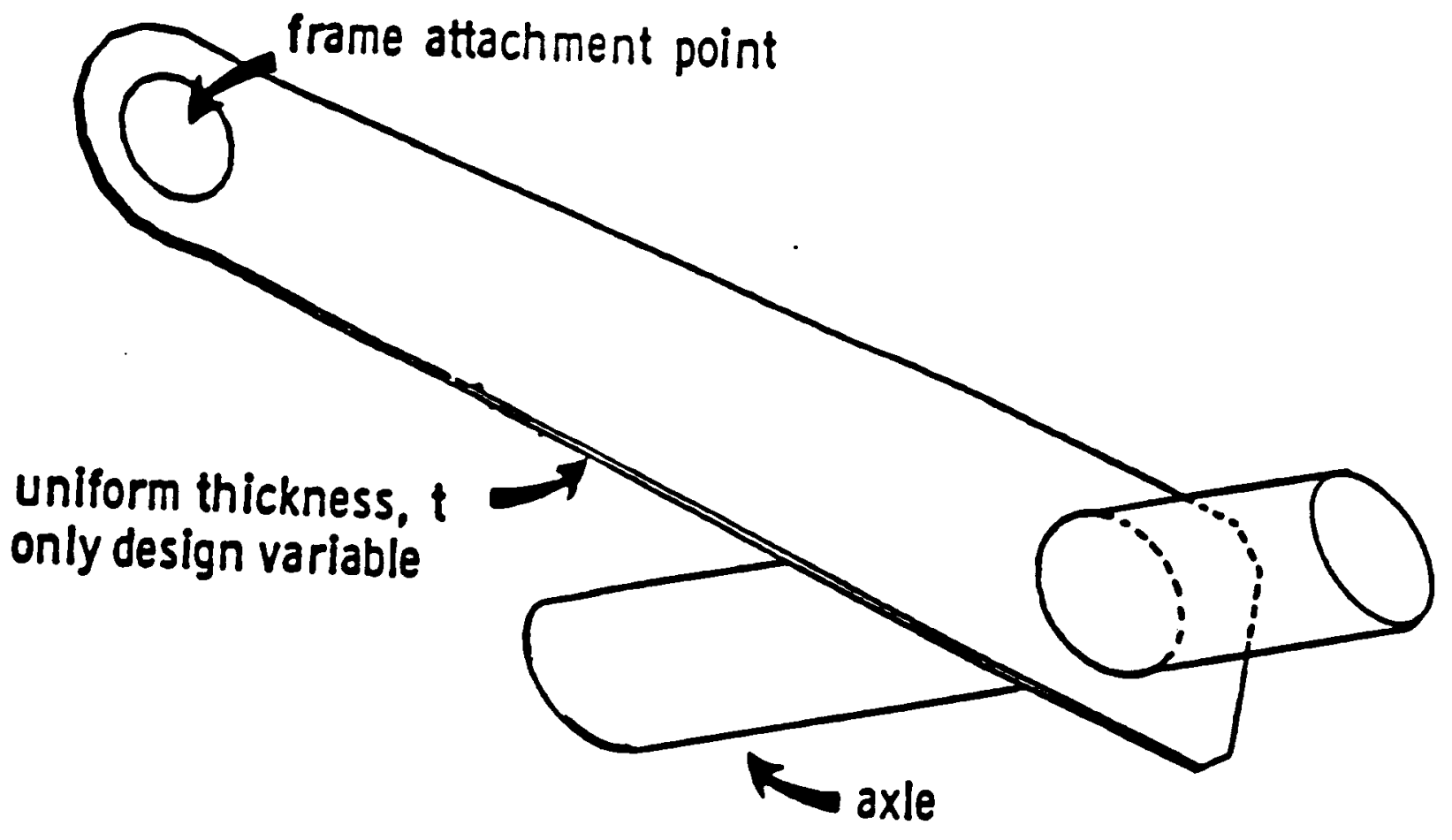

FIG. I Typical Part 
(3)

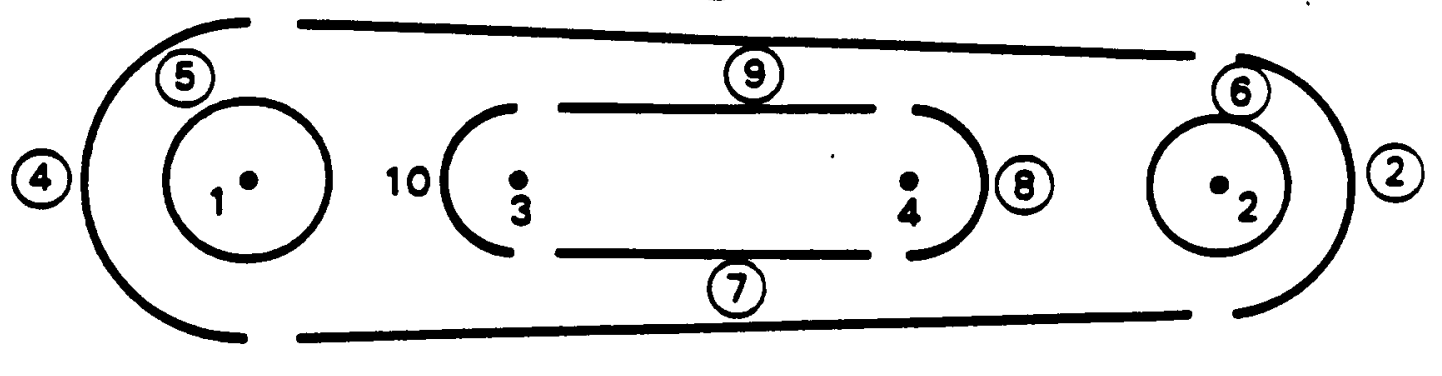

(1)

i Key Nodes
(i) Boundary Elements

FIG. 2 Boundary Elements For Typical Part 


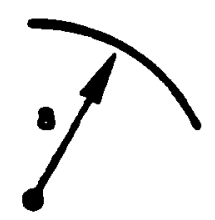

$$
\text { Key Node }
$$

a. Circular Are

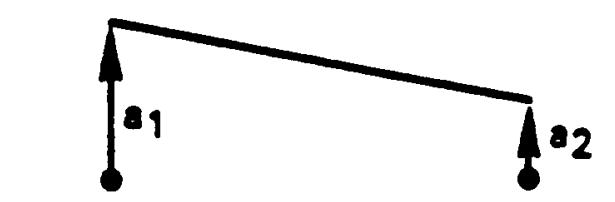

$$
\text { Key Node } 1 \quad \text { Key Node } 2
$$

b. Straight Line Segment

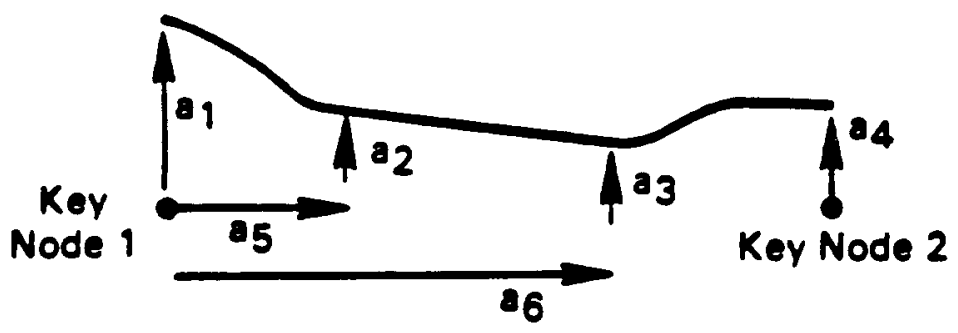

c. Double Cubic Connected by Straight Line.

\section{FIG. 3 Boundary Design Elements}




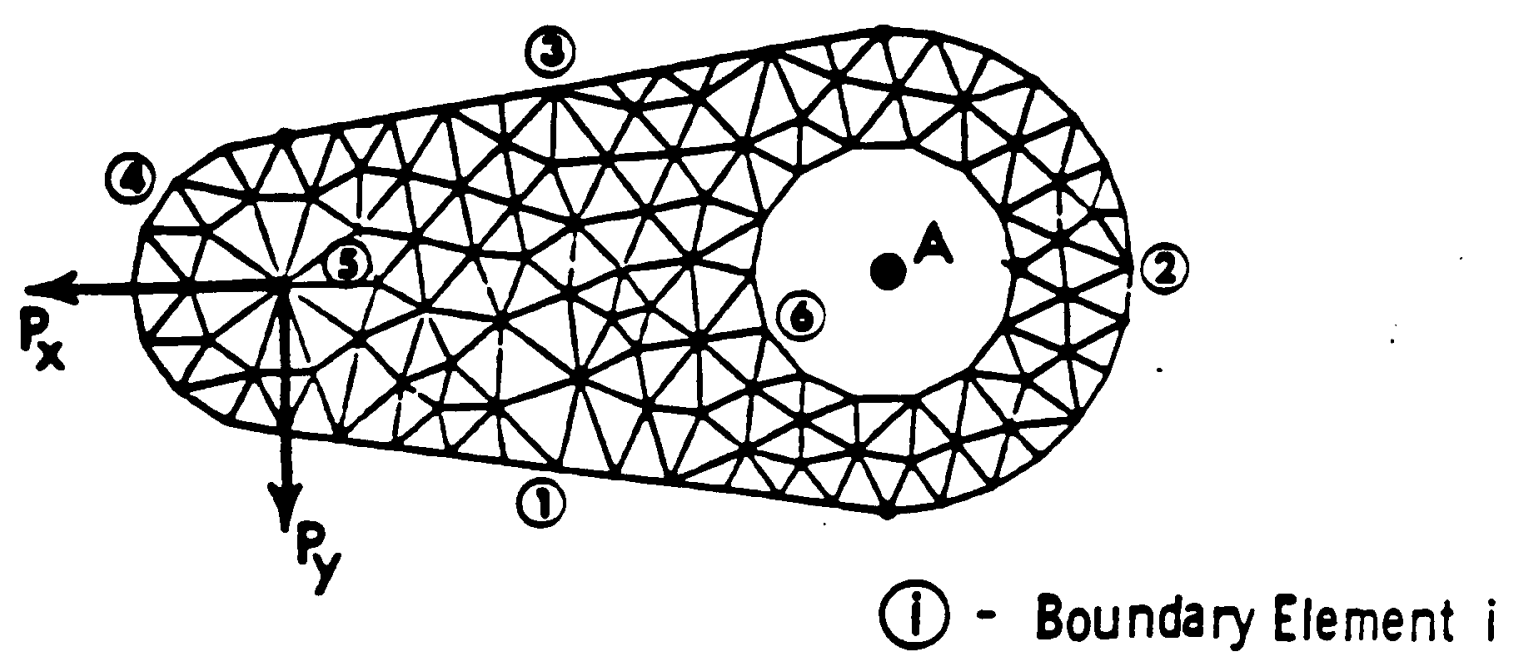

a) Initial Uniform Mesh

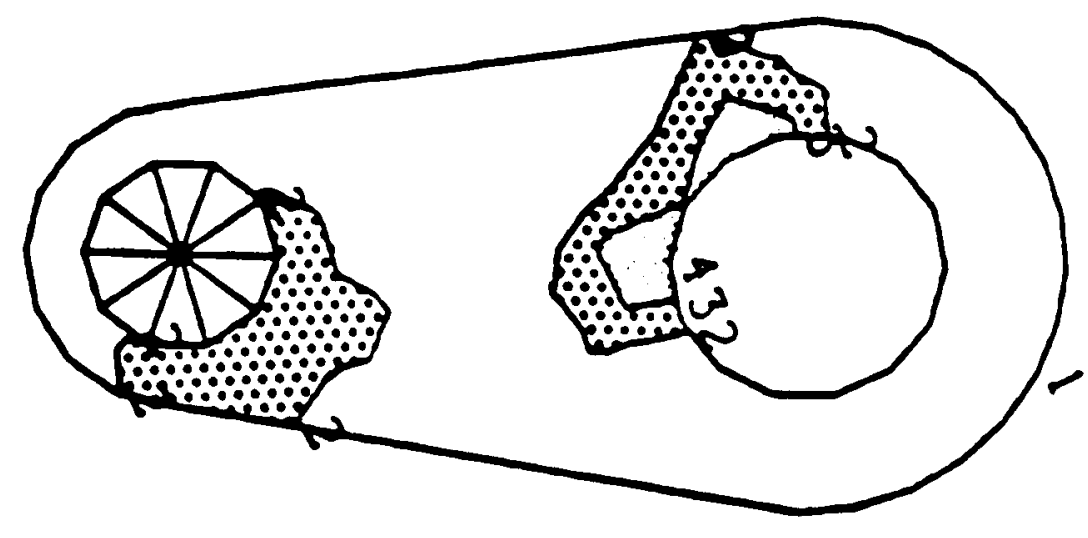

b) SED DIFFERENCE CONTOURS

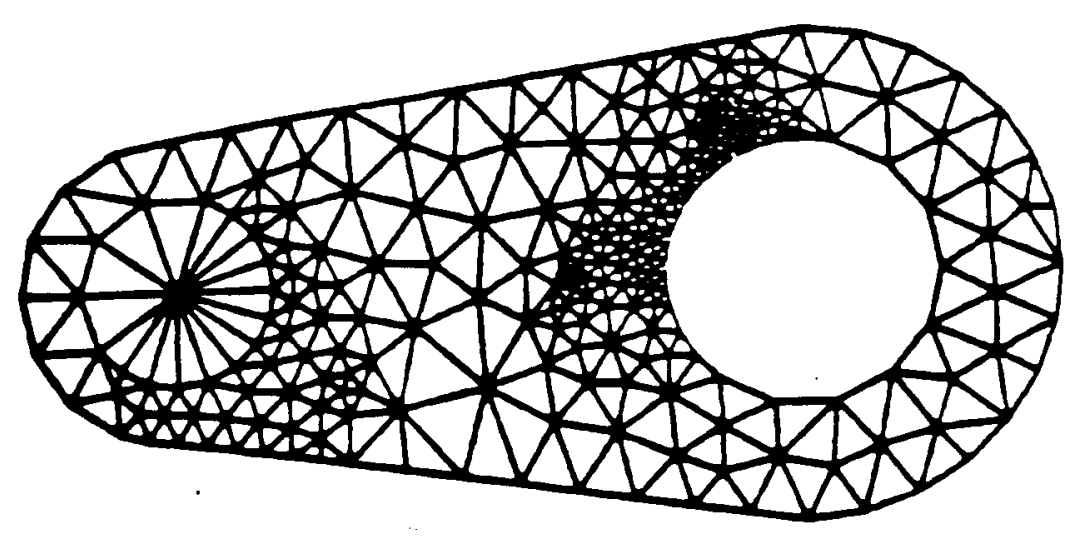

c) REFINED MESH

FIG. 4 Mesh Refinement 


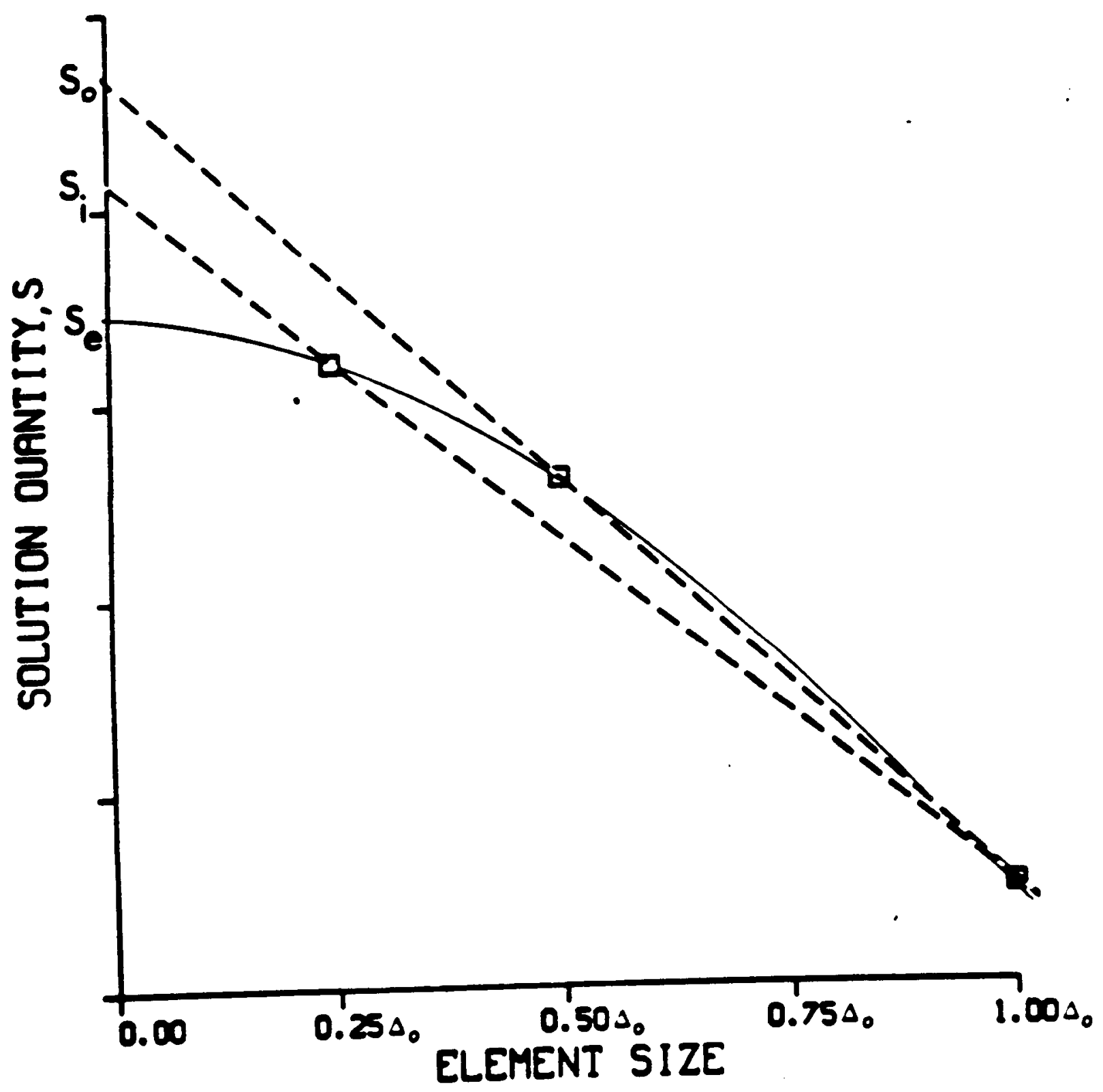

FIG. 5 TYPICAL SOLUTION CONVERGENCE 
(c) Projection
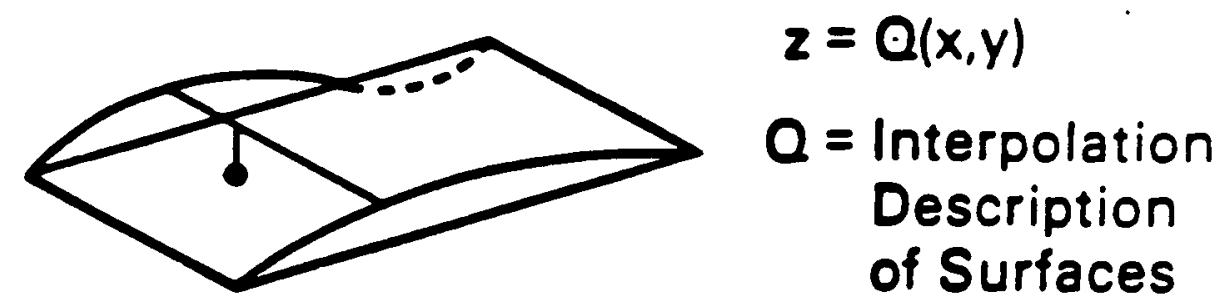

(b) Transformation

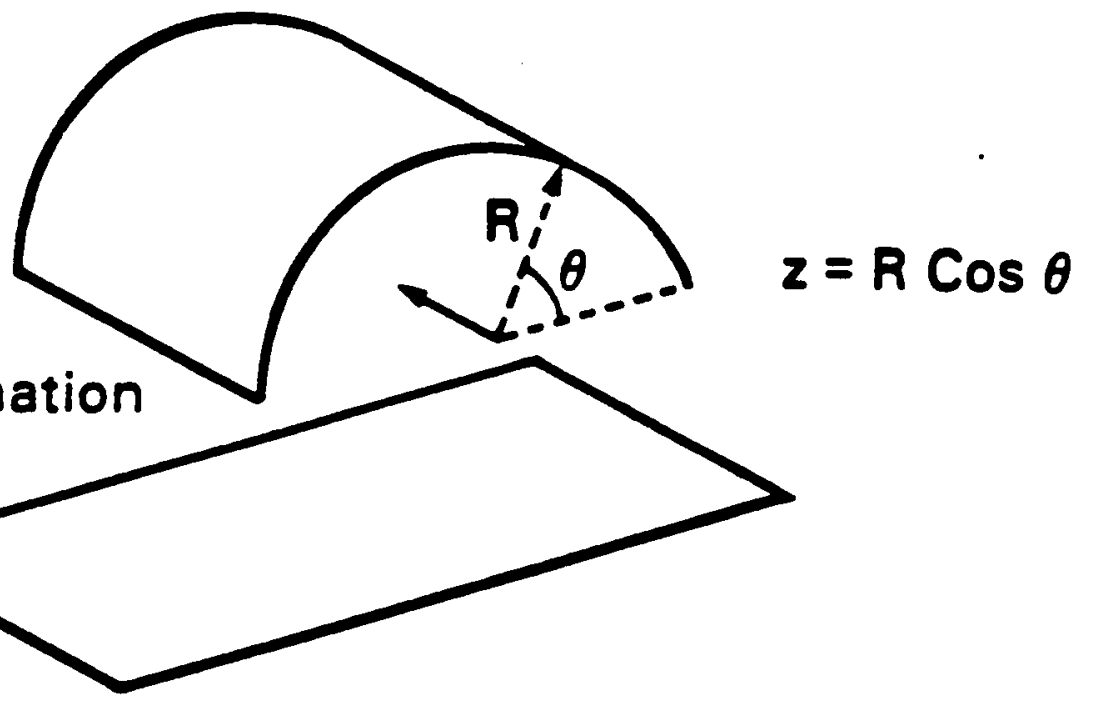

(a) Assembly of Segments

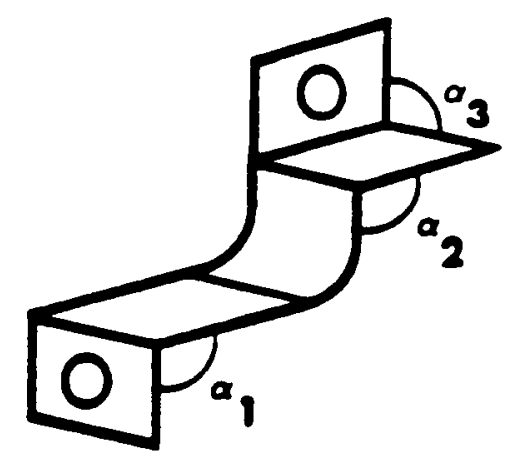

FIG. 6 THREE FORMS OF NON-PLANAR STRUCTURES 

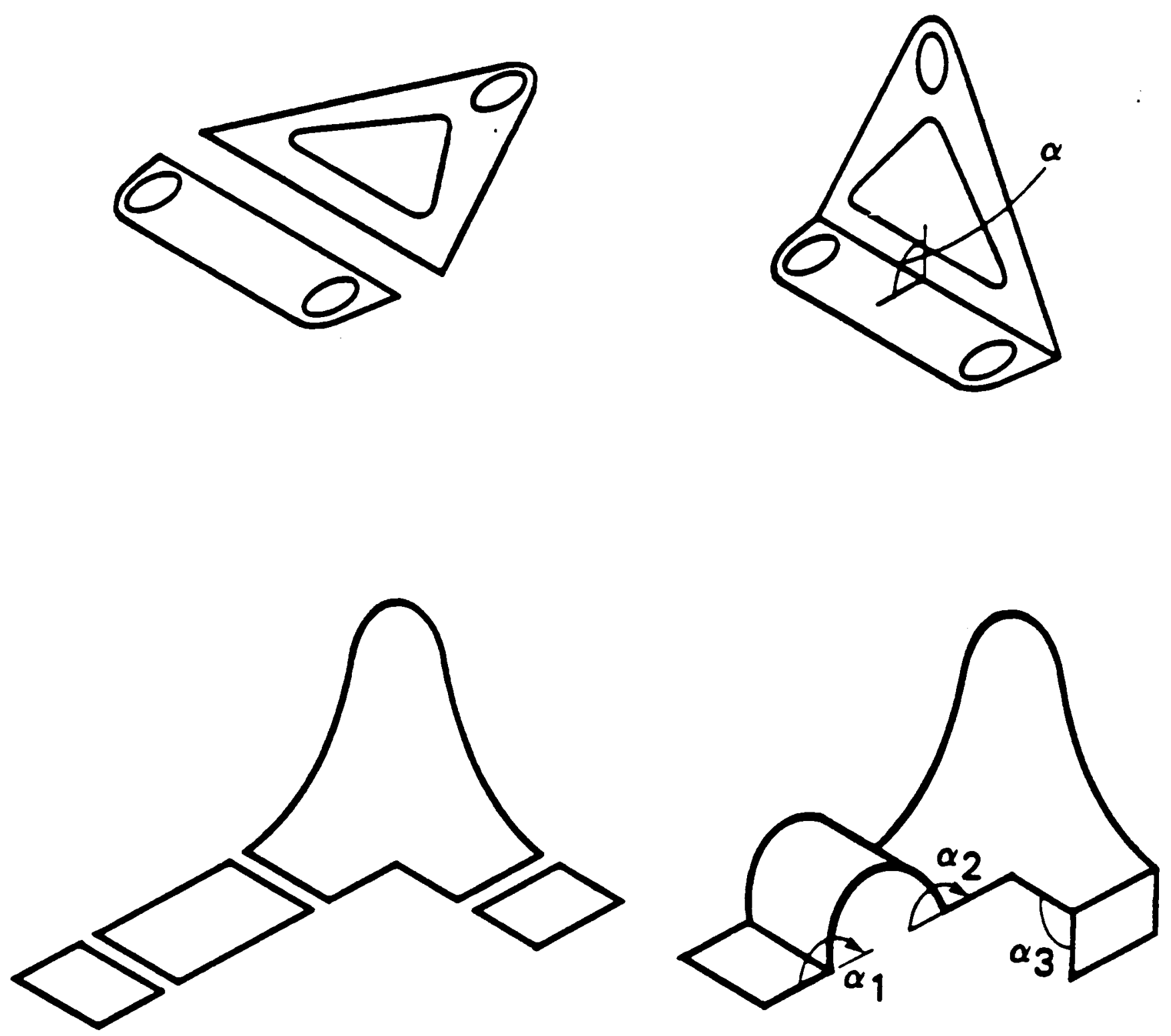

FIG. 7 Assembly and Rotation of Segments 


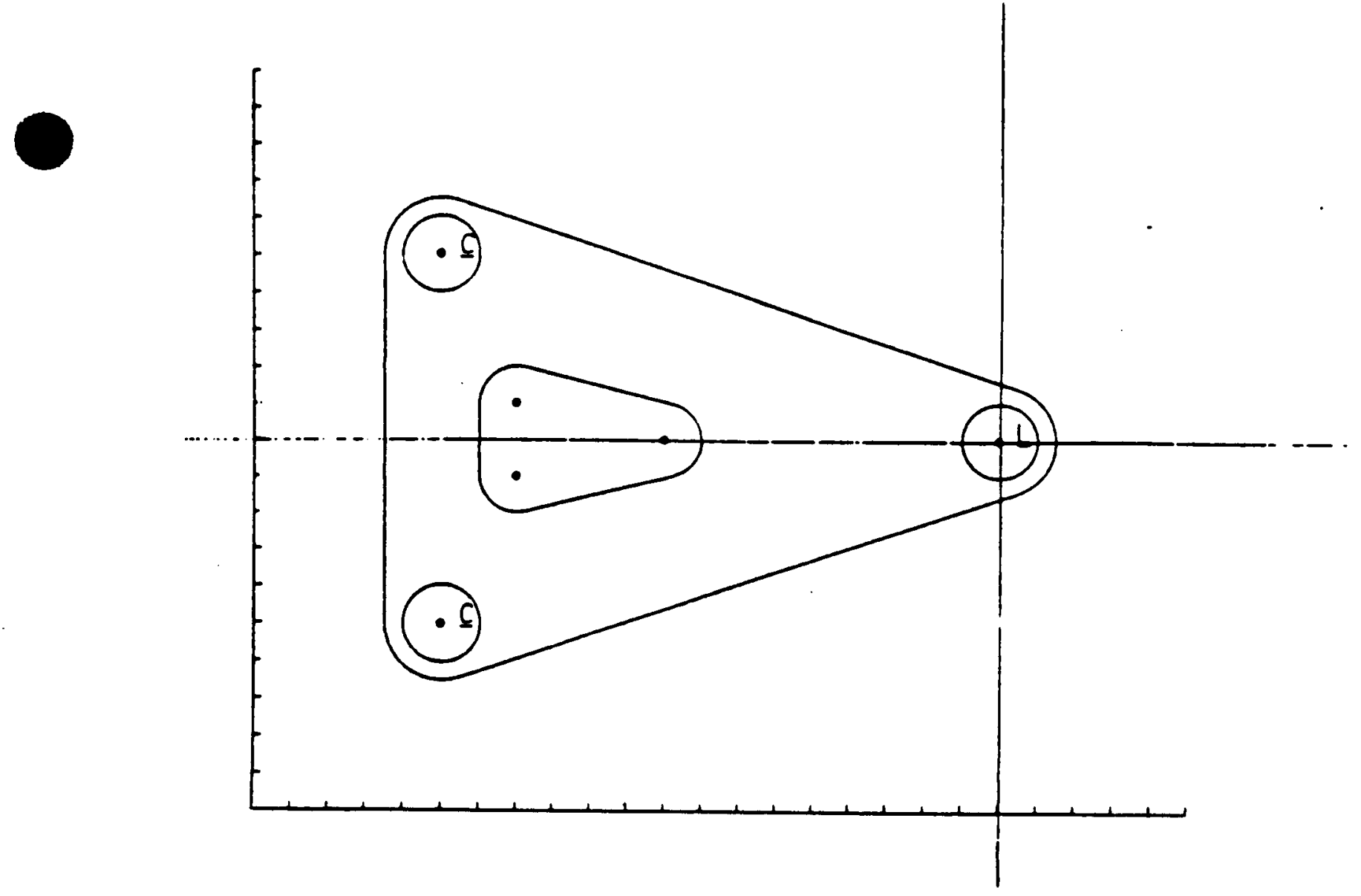

(b)Boundary Design Element Creation

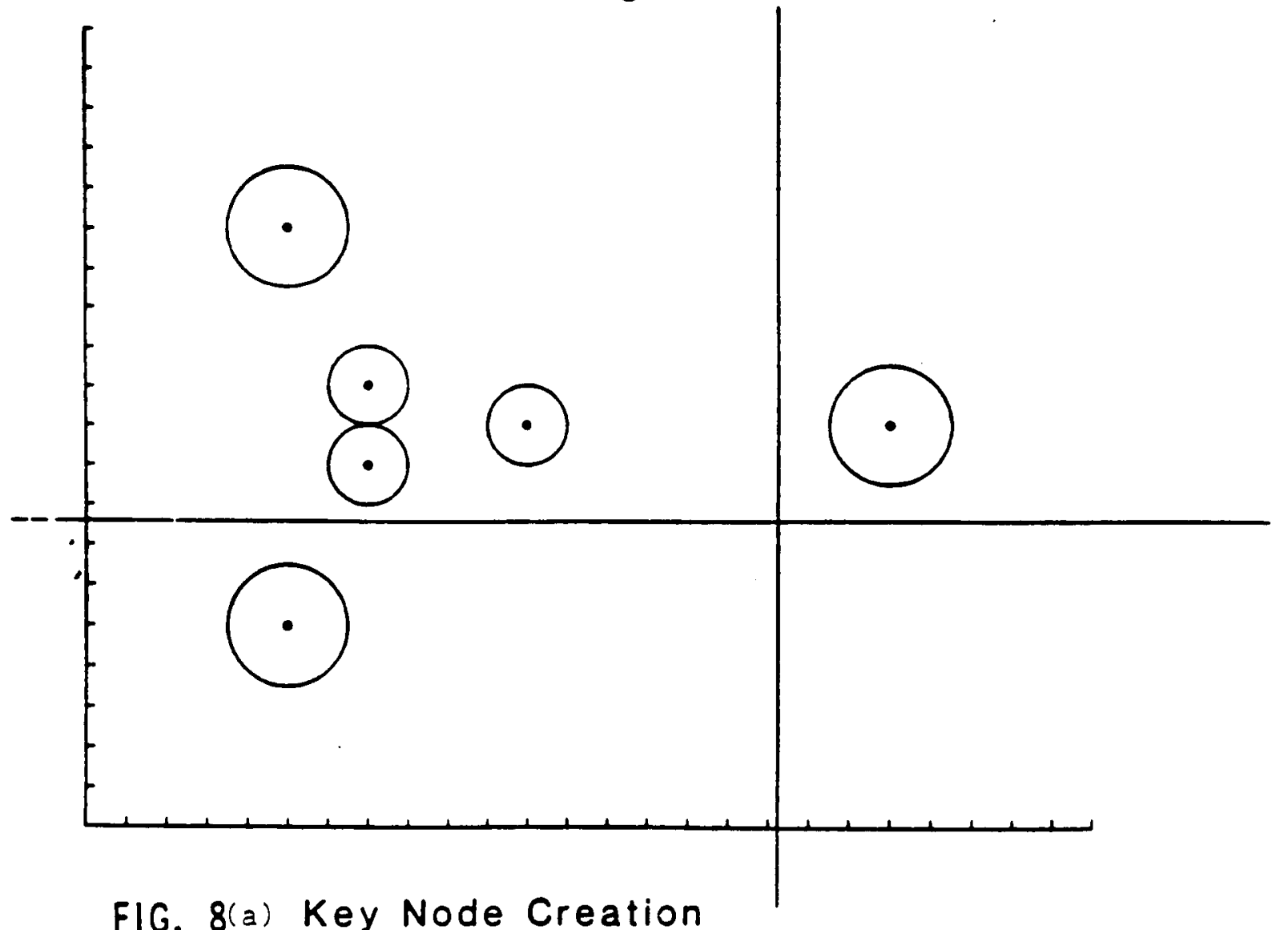




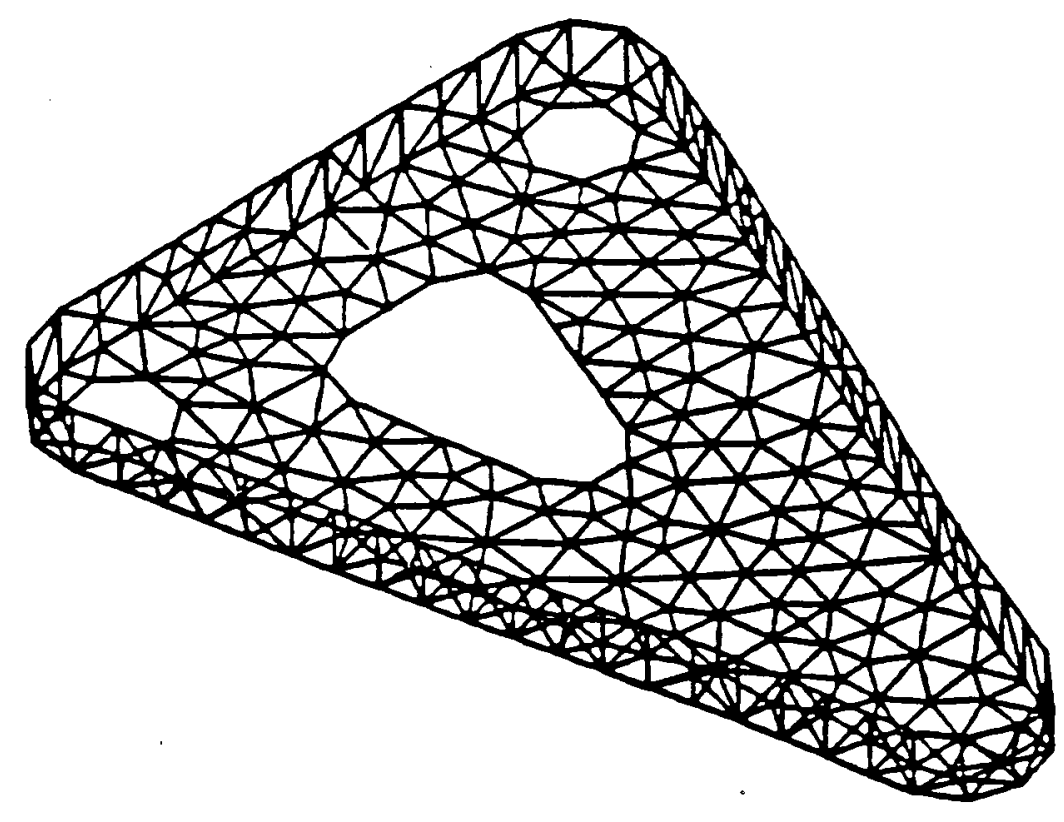

(b) Triangular Bracket Finite Element Mesh

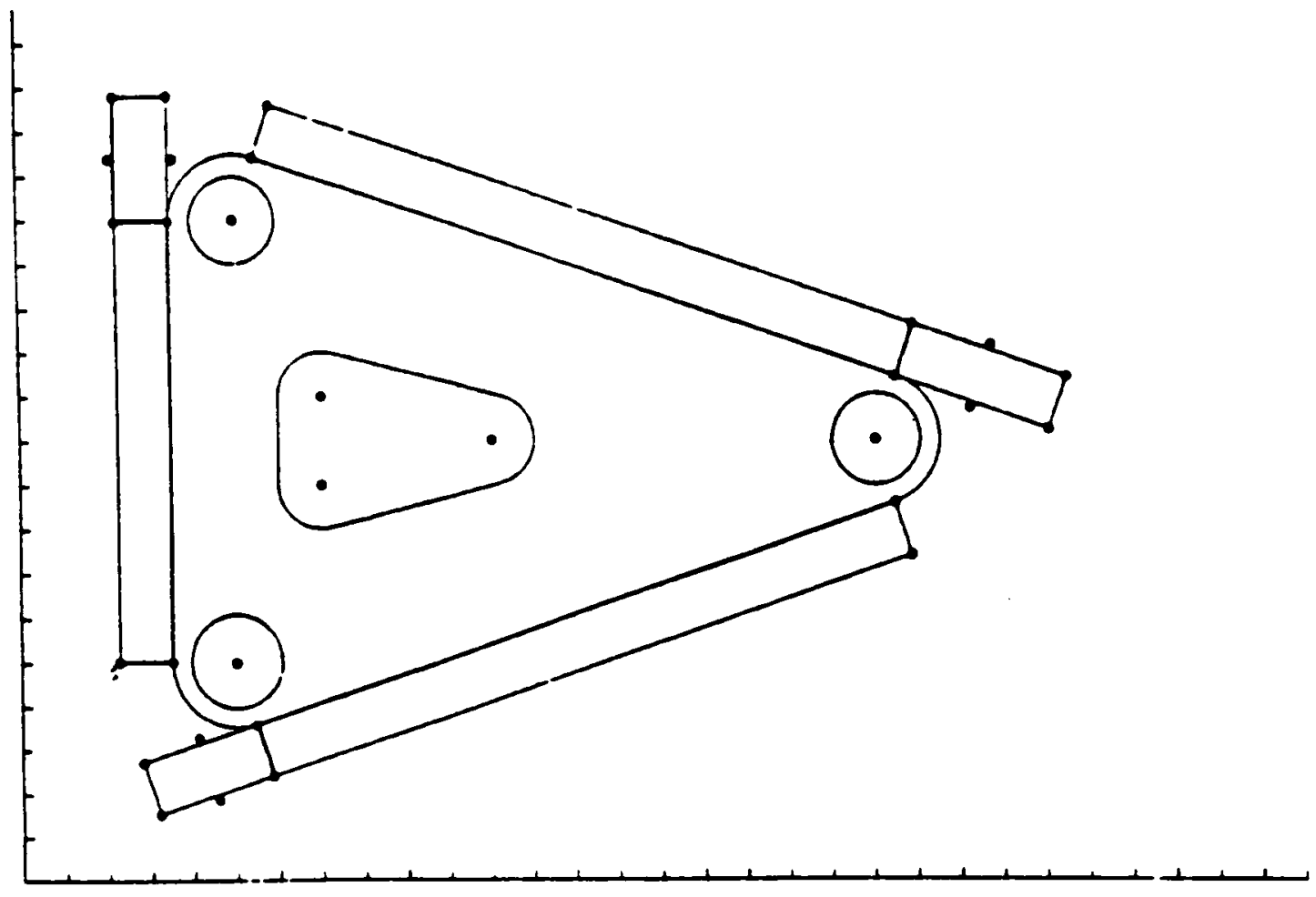

FIG. 9(a) Flanges Added to Triangular Bracket Model 


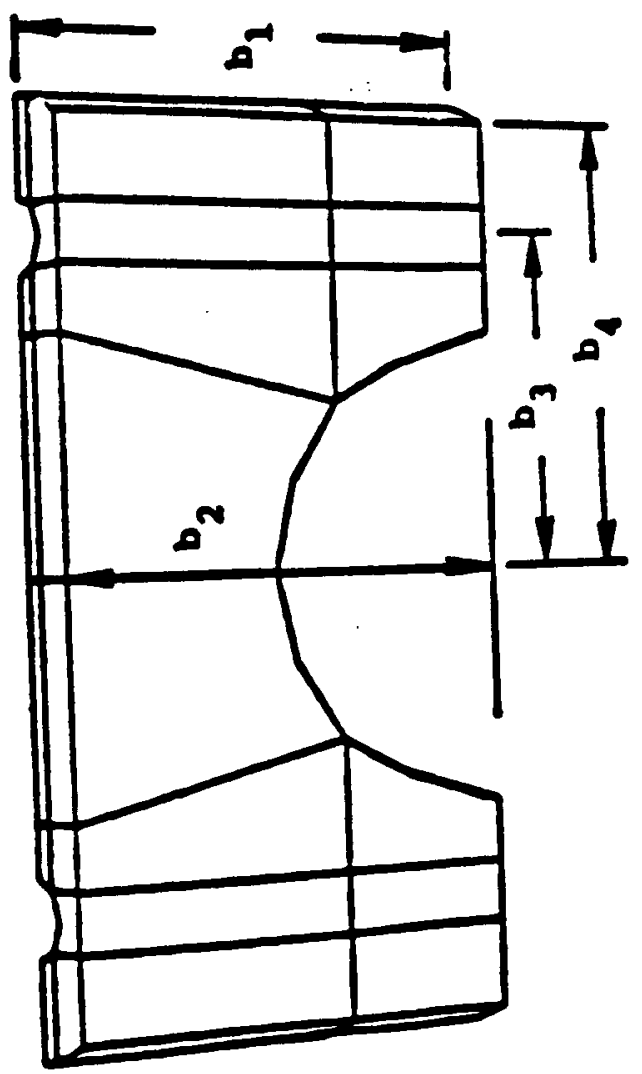

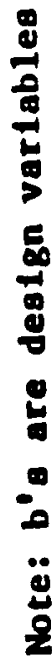

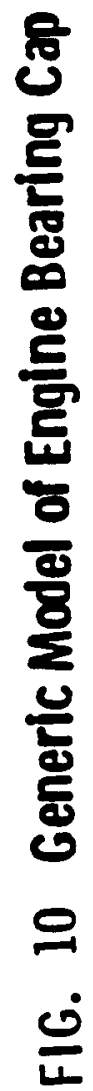




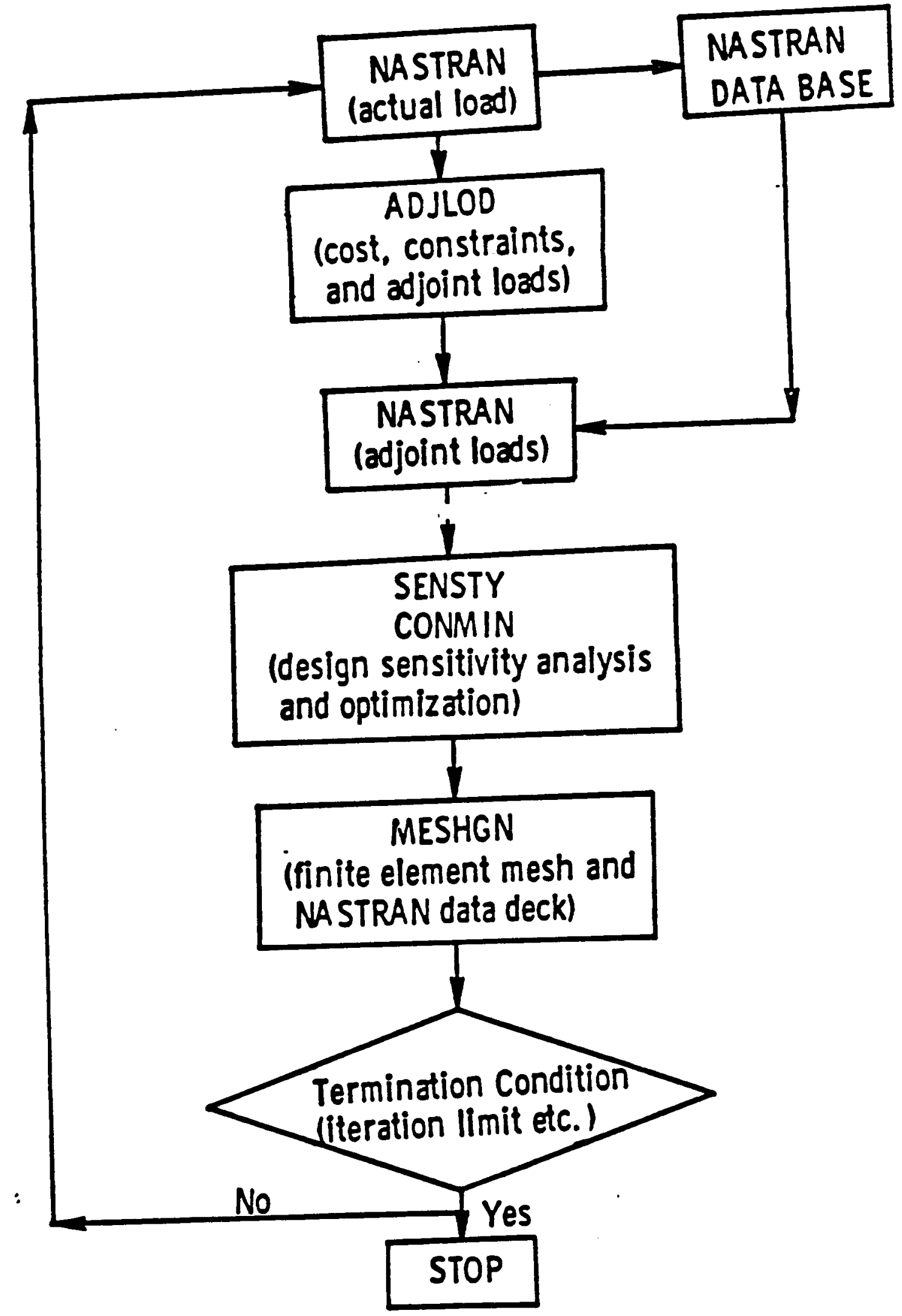

FIG. Il Flow Chart of Modularized System 
All Holes Have Radius of $1 \mathrm{~cm}$

All Dimensions in $\mathrm{cm}$

(i) - Design Element Number

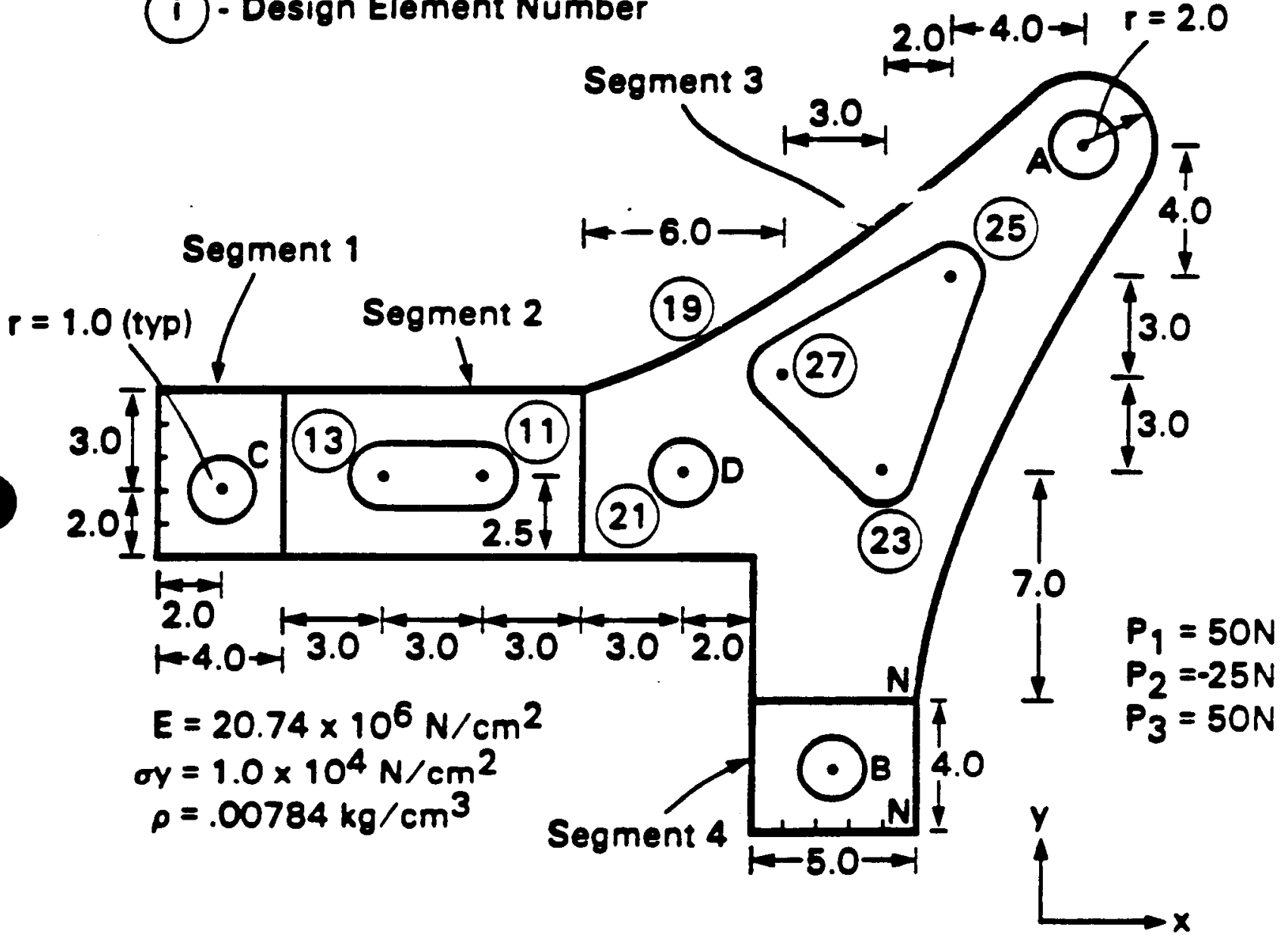

FIG. 12 DIMENSINNS OF EXAMPLE 

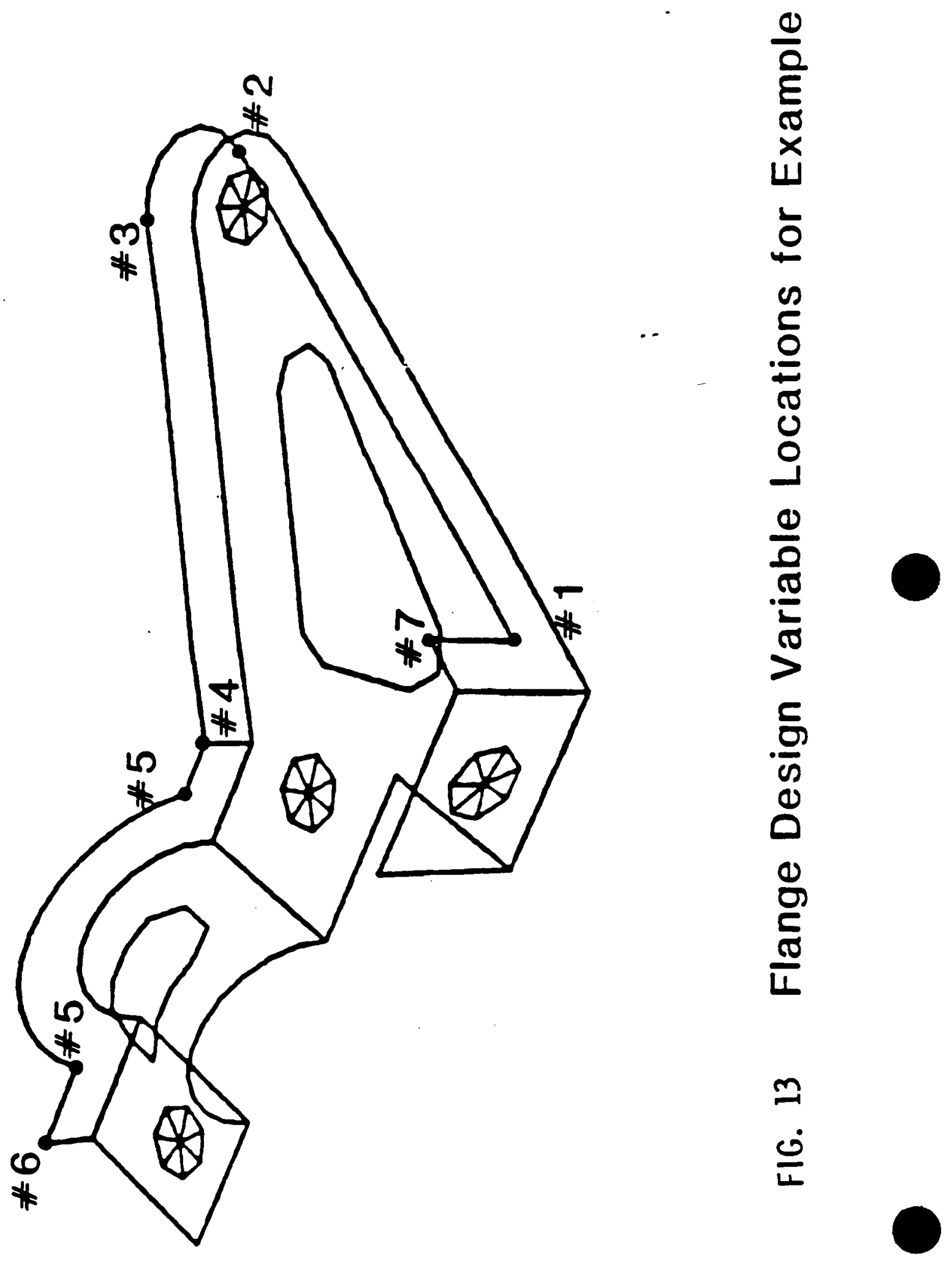


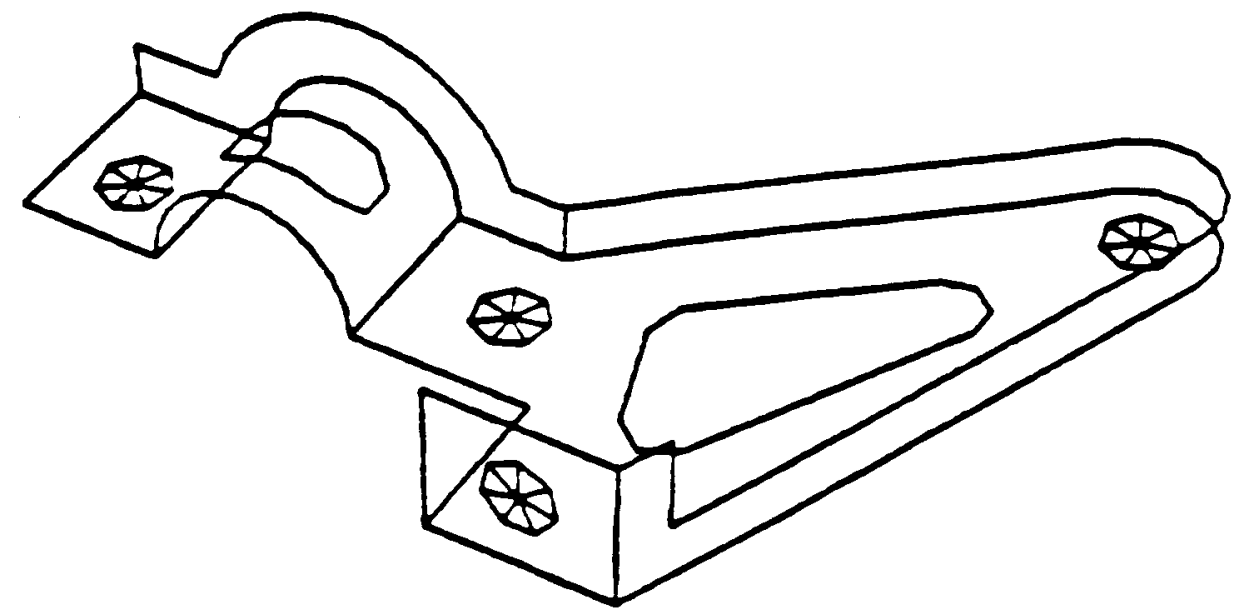

INITIAL DESIGN

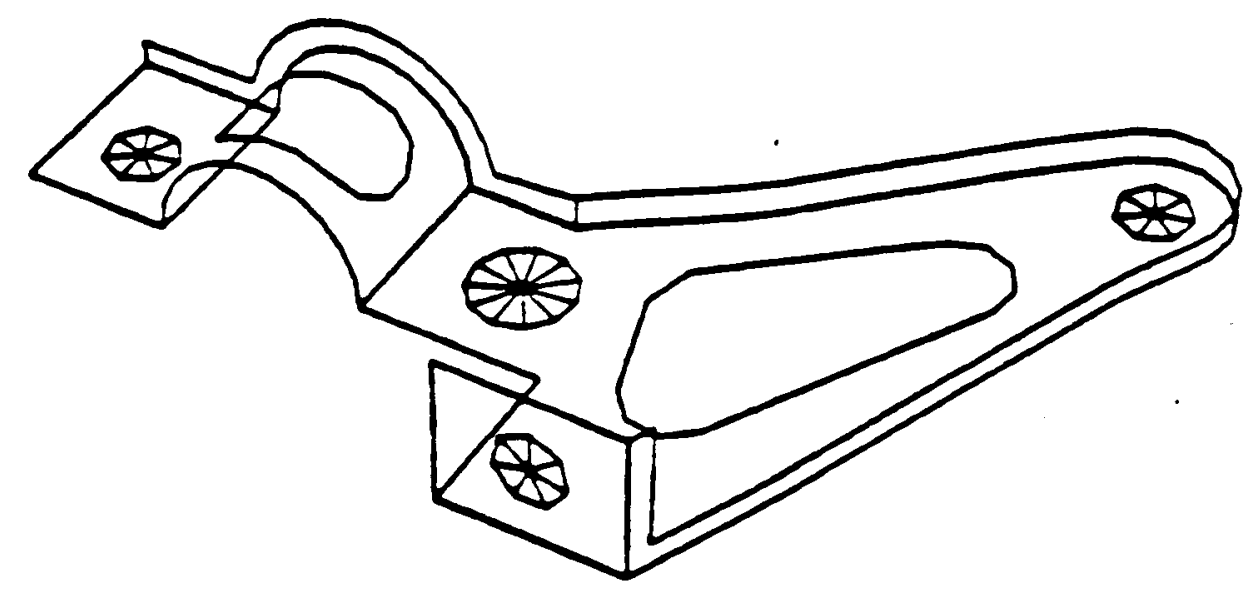

FINAL DESIGN

FIG. 15 Initial and Final Designs for Example 


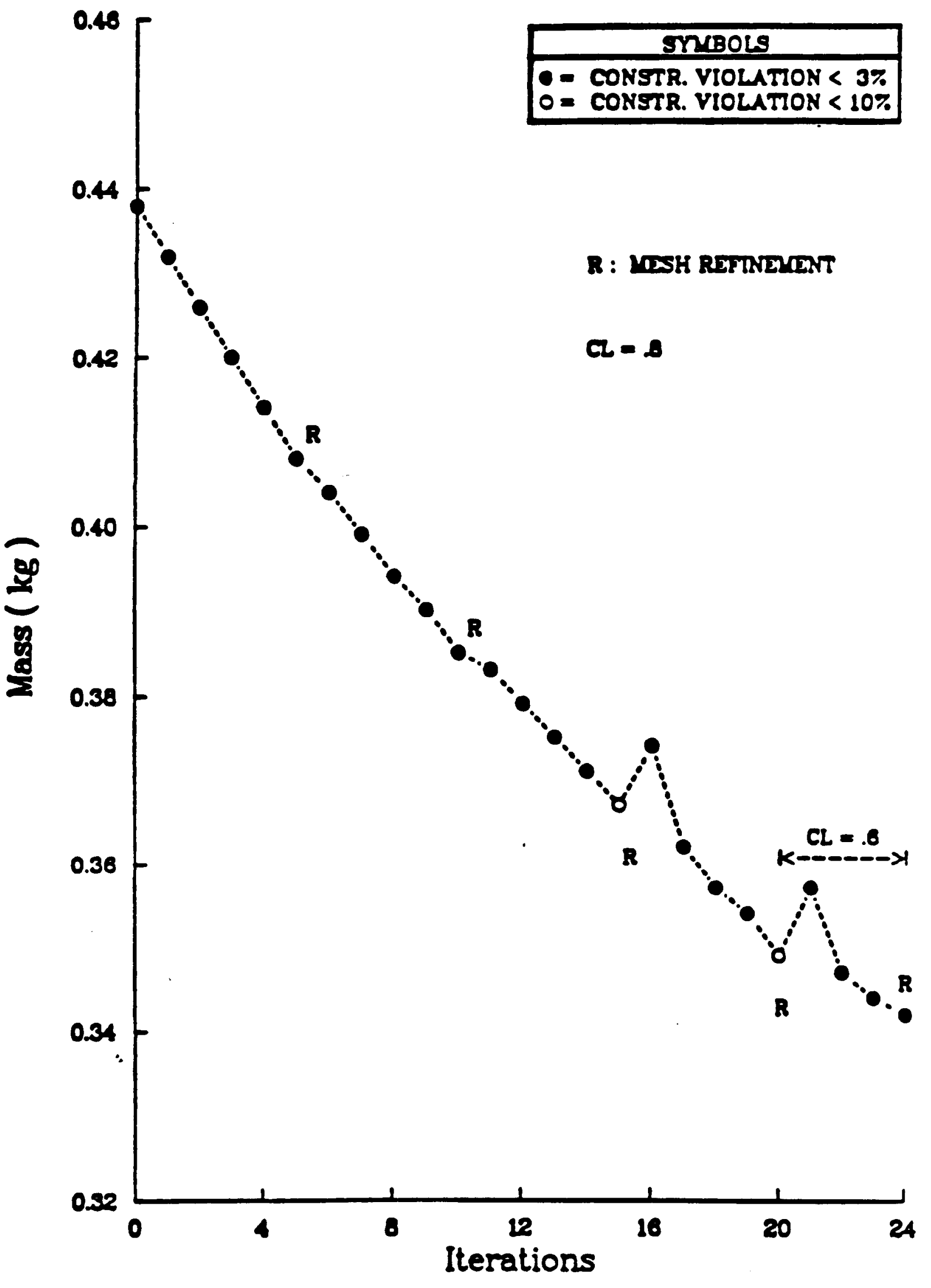

FIG. 16 Design History for Example 


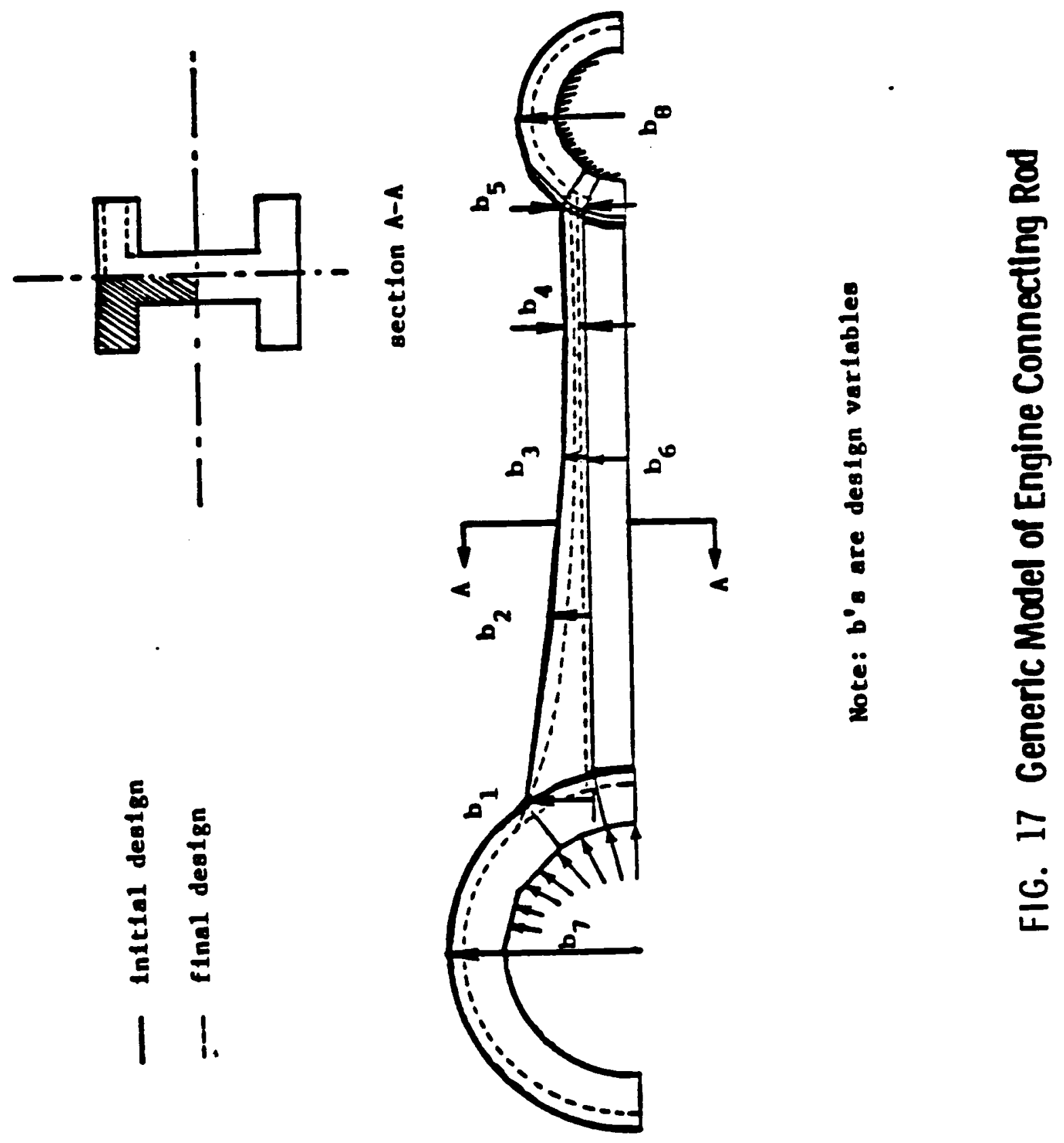





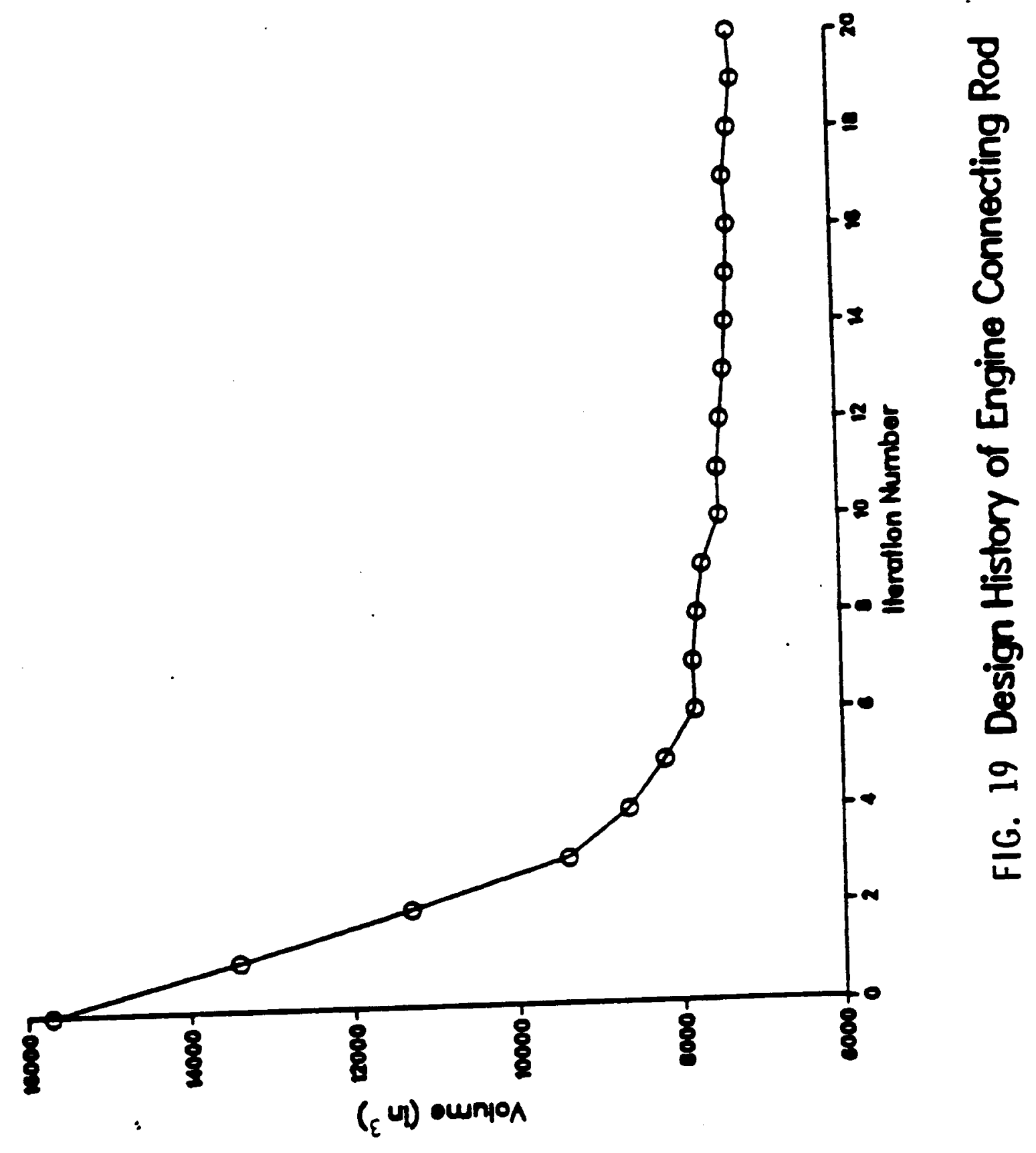




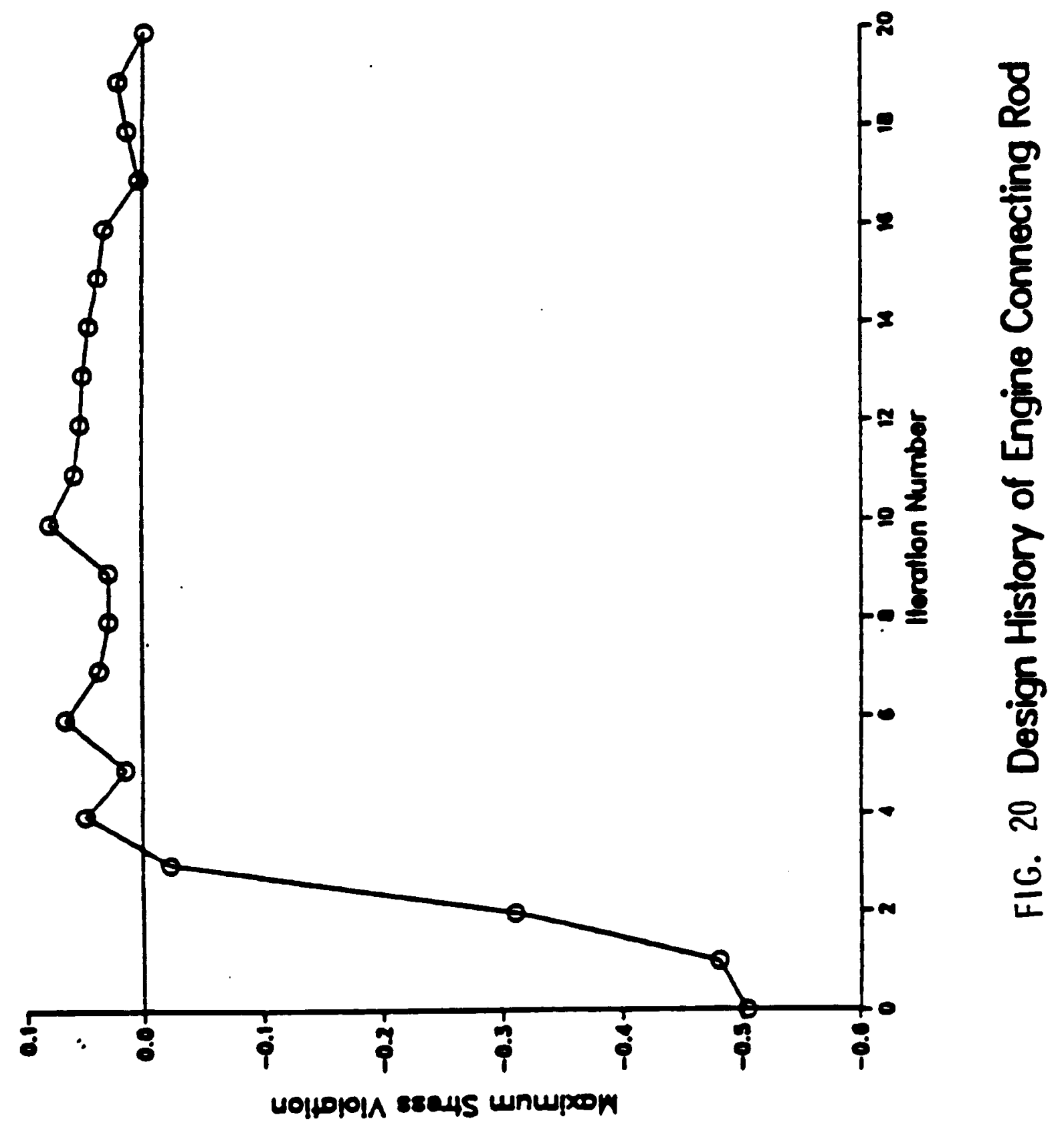

